Tadashi Ogawa and Hidehiko Komatsu

J Neurophysiol 101:721-736, 2009. First published Dec 10, 2008; doi:10.1152/jn.90817.2008

You might find this additional information useful...

This article cites 58 articles, 35 of which you can access free at:

http://jn.physiology.org/cgi/content/full/101/2/721\#BIBL

Updated information and services including high-resolution figures, can be found at:

http://jn.physiology.org/cgi/content/full/101/2/721

Additional material and information about Journal of Neurophysiology can be found at: http://www.the-aps.org/publications/jn

This information is current as of February 10, 2009.

Journal of Neurophysiology publishes original articles on the function of the nervous system. It is published 12 times a year (monthly) by the American Physiological Society, 9650 Rockville Pike, Bethesda MD 20814-3991. Copyright (C) 2005 by the American Physiological Society. ISSN: 0022-3077, ESSN: 1522-1598. Visit our website at http://www.the-aps.org/. 


\title{
Condition-Dependent and Condition-Independent Target Selection in the Macaque Posterior Parietal Cortex
}

\author{
Tadashi Ogawa ${ }^{1,2}$ and Hidehiko Komatsu ${ }^{1}$ \\ ${ }^{1}$ Division of Sensory and Cognitive Information, National Institute for Physiological Sciences, Aichi; and ${ }^{2}$ Department of Integrative Brain \\ Science, Graduate School of Medicine, Kyoto University, Kyoto, Japan
}

Submitted 28 July 2008; accepted in final form 4 December 2008

\begin{abstract}
Ogawa T, Komatsu H. Condition-dependent and condition-independent target selection in the macaque posterior parietal cortex. $J$ Neurophysiol 101: 721-736, 2009. First published December 10, 2008; doi:10.1152/jn.90817.2008. During a visual search, information about the visual attributes of an object and associated behavioral requirements is essential for discriminating a target object from others in the visual field. On the other hand, information about the object's position appears to be more important when orienting the eyes toward the target. To understand the neural mechanisms underlying such a transition (i.e., from nonspatial- to spatial-based target selection), we examined the dependence of neuronal activity in the macaque posterior parietal cortex (PPC) on visual sensory properties and ongoing task demands. Monkeys were trained to perform a visual search task in which either a shape or color singleton within an array was the target, depending on the ongoing search dimension. The visual properties and the task demands were manipulated by independently changing the stimulus features (shape and color), singleton type, and search dimension. We found that a subset of PPC neurons significantly discriminated the target from other stimuli only when the target was defined by a particular stimulus dimension and had specific stimulus features, such as a shape-singleton, bar stimulus (conditiondependent target selection), whereas another subset did so irrespective of the stimulus features and the target-defining dimension (conditionindependent target selection). There was thus a great deal of variety in the neural representations specifying the locus of the target. The coexistence of these distinctly different types of target-discrimination processes suggests that the PPC may be situated at the level where the transition from nonspatial- to spatial-based target selection takes place.
\end{abstract}

\section{N T R O D U C T I O N}

During a visual search, information about the visual sensory properties of individual objects and the ongoing behavioral requirements is essential for discriminating an object of interest from others in the visual field. On the other hand, information about the locus of the target is more important when shifting covert spatial attention and overt eye movement toward it. This information flow may correspond to the transition from nonspatial- to spatial-based target-selection processes. In fact, neuronal activity in the visual areas richly represents the sensory properties of objects and is influenced not only by spatial attention but also by feature-based attention (e.g., Bichot et al. 2005; Motter 1994). By contrast, activity in visualmotor areas, such as the frontal eye field (FEF) and the superior colliculus, is less sensitive to the sensory properties of objects (McPeek and Keller 2002; Mohler et al. 1973; Pigarev et al.

\footnotetext{
Address for reprint requests and other correspondence: T. Ogawa, Department of Integrative Brain Science, Graduate School of Medicine, Kyoto University, Sakyo-ku 606-8501, Kyoto, Japan (E-mail: togawa@brain.med.kyoto-u.ac.jp).
}

1979; but see Peng et al. 2008) and is preferentially related to spatial attention (Kodaka et al. 1997; Monosov et al. 2008; Moore and Fallah 2004; Thompson et al. 2005).

Several lines of evidence suggest the importance of the posterior parietal cortex (PPC) in the transition from nonspatial- to spatial-based target selection. On the one hand, area $7 \mathrm{a}$ and the lateral intraparietal area (LIP), which are visual-motor areas (Barash et al. 1991a,b; Gnadt and Andersen 1988), play crucial roles in target selection (Buschman and Miller 2007; Constantinidis and Steinmetz 2001; Thomas and Paré 2007; Wardak et al. 2002), spatial attention and anticipation (Bisley and Goldberg 2003; Bushnell et al. 1981; Colby et al. 1996; Duhamel et al. 1992), and saccade planning (Snyder et al. 1997). On the other hand, the activity of PPC neurons can directly reflect visual sensory attributes, such as the color and shape of a stimulus (Durand et al. 2007; Konen and Kastner 2008; Sereno and Amador 2006; Sereno and Maunsell 1998; Toth and Assad 2002). Thus the PPC may be situated such that it is able to play a key role in the transition from nonspatial- to spatial-based target selection.

To obtain insight into the neural mechanism underlying such a transition process during target selection, we examined the dependence of neuronal activity in the PPC of the macaque on visual sensory properties and ongoing task demands. Monkeys were trained to perform a visual search task in which two singleton stimuli, unique in their color or shape dimension, were presented within an array. The monkeys had to make a saccade to the singleton that was unique in the target-defining dimension. The sensory conditions of the receptive field stimulus and the task demand were manipulated by independently changing stimulus features, singleton type, and search dimension. One advantage of this task design is that we are able to assess not only whether the activity related to the target selection is dependent on the stimulus features but also whether it is dependent on the stimulus dimension defining the target. Using this task, we previously found that most V4 neurons significantly discriminated the target from the other stimuli only when the target had specific stimulus features and was defined by one particular stimulus dimension, whereas FEF neurons discriminated the target irrespective of the stimulus features and target-defining dimension (Ogawa and Komatsu 2004, 2006). The current results demonstrated that both types of neurons existed in the PPC, suggesting that the PPC might be situated at the stage where the transition from nonspatial- to spatial-based target selection takes place.

\footnotetext{
The costs of publication of this article were defrayed in part by the payment of page charges. The article must therefore be hereby marked "advertisement" in accordance with 18 U.S.C. Section 1734 solely to indicate this fact.
} 


\section{METHODS}

\section{Subjects and surgery}

Two Japanese macaque monkeys (Macaca fuscata, monkeys Q and $\mathrm{L}$, both male and weighing 5.8 and $7.5 \mathrm{~kg}$, respectively) were used in this study. Surgery was performed on each monkey while the animal was under deep anesthesia (intravenous sodium pentobarbital) and an eye coil, head holder, and recording chambers were implanted using sterile techniques. Using magnetic resonance (MR) images obtained prior to surgery, the recording chambers were placed at stereotaxic coordinates over the PPC. They were anchored flat against the skull so that recording electrodes could be advanced along the bank of the intraparietal sulcus. The monkeys were allowed to recover for $>2 \mathrm{wk}$ before initiation of training and recording. During the training and recording periods, the monkeys were deprived of water on weekdays, although their weight and health status were carefully monitored, and they were given additional water or food when necessary. All procedures for animal care and the experimental protocols were in accordance with the National Institutes of Health Guidelines for the Care and Use of Laboratory Animals (1996) and were approved by the Institutional Animal Experimentation Committee.

\section{Visual stimuli and behavioral tasks}

In the present study, the monkeys were required to perform a multidimensional visual search task in which an array of six stimuli appeared on an imaginary circle around a central fixation point, all separated by the same distance (Fig. 1). In each array, two singletons, one unique in the shape dimension (shape singleton), the other in the color dimension (color singleton), were presented with four additional identical stimuli (nonsingleton). Each stimulus was made from two possible shapes (bar and circle) and two possible colors (cyan and yellow). One of the singletons served as a target and the other as a distractor, depending on the ongoing target-defining dimension, which was indicated by the color of the fixation spot (shape search, red; color search, blue). In the shape search, the shape singleton was the target and the color singleton was the distractor (Fig. 1, top row)

\section{Singleton type}

Shape singleton Color singleton

Nonsingleton
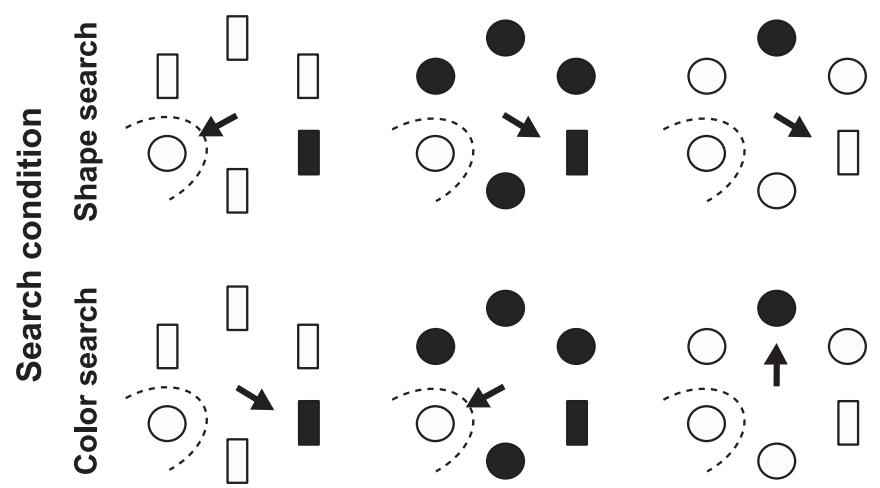

FIG. 1. Experimental paradigm and visual stimuli. Stimulus conditions for the experiment. Two singleton stimuli, one unique in the color dimension, the other in the shape dimension, were presented simultaneously with four additional identical stimuli. Open and filled symbols correspond to cyan and yellow, respectively. Monkeys had to make a saccade (arrows) to one of the singleton stimuli, depending on the instructed target-defining dimension. In the shape search (top row), the shape singleton was the target and the color singleton was the distractor. In the color search, the shape singleton was the distractor and the color singleton was the target. The nonsingleton stimulus never became the target (nontarget). Examination of the 2 search conditions was conducted in separate blocks. The ongoing target-defining dimension was signaled by the color of the fixation spot. and the opposite was the case in the color search (Fig. 1, bottom row). A nonsingleton never became the target under either search condition (this stimulus condition will be referred to as nontarget, in contrast to the target and distractor conditions). Note that one of the three behavioral relevance conditions (target, distractor, and nontarget) corresponded to each of the three singletontypes (shape singleton, color singleton, and nonsingleton), although the linkages between the behavioral relevance conditions (target and distractor) and the singleton-type conditions (shape and color singletons) alternated when the search dimension was switched.

Identical sets of 13 different arrays were presented under each search condition. Of these, one array was used in a catch trial in which all six stimuli had the same color and shape. In that case, the monkeys were given a reward when they held fixation throughout the trial (data from catch trials were excluded from the present analysis). The remaining 12 arrays were used in visual search trials (two shapes $\times$ two colors $x$ three behavioral relevance conditions). Thus with the two search conditions (shape search and color search), in all there were 24 different trial conditions. The target, distractor, and nontarget stimuli were presented with the same frequency at three possible positions: one within the receptive field and two $120^{\circ}$ from the receptive field in either the clockwise or counterclockwise direction (the conditions under which the target appeared in the receptive field will be referred to as the target conditions). At the remaining three positions, nontarget (nonsingleton) stimuli were always presented. Stimulus shape and color were randomly selected from the two possible shapes (bar and circle) and the two possible colors (cyan and yellow) and were also presented with the same frequency at all six possible positions.

Each trial began with the onset of a fixation spot at the center of a monitor screen. The monkeys had to fixate on that spot within a window of $\pm 0.75-1.0^{\circ}$. After fixation for $800-1,500 \mathrm{~ms}$ (typically $1,200 \mathrm{~ms}$, although it varied among neurons), the array was displayed. The monkeys were then required to make a saccade to the target. There was no artificial delay period (i.e., it was reaction-time task). When a monkey made a single saccade landing inside a square window around the target whose size was $35 \%$ of the diameter of the array, it received a liquid reward. If the gaze deviated from the fixation window before the array was presented, or if the saccade latency was too short $(<120 \mathrm{~ms})$, the trial was aborted immediately without reward and neuronal data were not recorded. For each neuron, the shape search and color search conditions were applied sequentially in separate trial blocks (each block typically consisted of 78 trials $=13$ conditions $\times 6$ repetitions) and each search condition was repeated more than twice (i.e., a total of $>12$ repetitions of each trial condition, mean $=16.1$ repetitions). The target-defining dimension used in the initial block was randomly selected.

Visual stimuli were generated using a video signal generator (VSG 2/3; Cambridge Research Systems, Cambridge, UK) and presented on a video monitor with a $120-\mathrm{Hz}$ refresh rate and $800 \times 600$ resolution (GDM-2000TD; Sony, Tokyo, Japan). They were viewed binocularly from a distance of $65 \mathrm{~cm}$ in a dark room and subtended a visual angle of $30 \times 24^{\circ}$. All of the elements in each array were the same size and had the same luminance $\left(10 \mathrm{~cd} / \mathrm{m}^{2}\right)$. The background was uniformly dark gray with a luminance of $0.1 \mathrm{~cd} / \mathrm{m}^{2}$. The fixation point was a small spot subtending $0.1^{\circ}$. The square root of the stimulus area varied linearly depending on its distance from the fixation point (e.g., $1.0^{\circ}$ at $10^{\circ}$ eccentricity).

A memory-guided saccade task (Hikosaka and Wurtz 1983) was also used to detect delay-period and saccade-burst activity and to assess the extent of the receptive field. While the monkeys maintained fixation during this task, an isolated stimulus (luminance $=10 \mathrm{~cd} / \mathrm{m}^{2}$, size $=1 \mathrm{deg}^{2}$ ) was flashed for 200 or $500 \mathrm{~ms}$ at one of two or six peripheral positions on an imaginary circle centered at the fixation spot. After a delay of $800-1,500 \mathrm{~ms}$, the fixation point was removed and the monkeys were rewarded for making a saccade to the location of the previously flashed stimulus. 


\section{Data collection}

Recordings were made from three hemispheres of the two macaques (one hemisphere in monkey $\mathrm{Q}$ and two hemispheres in monkey L). Single neurons were recorded using a stainless steel or tungsten electrode (FHC, Bowdoinham, ME) having an impedance $>2.0 \mathrm{M} \Omega$ measured at $1 \mathrm{kHz}$ (Model IMP-1; Bak Electronics, Germantown, MD). Extracellular activity was amplified using a microelectrode AC amplifier (Model-1800; AM-Systems, Carlsborg, WA) and stored on a computer equipped with a multichannel A/D board at a sampling rate of $25 \mathrm{kHz}$ (PCI-416L2A; Datel Japan, Tokyo). Single units were discriminated according to spike amplitude using a simple threshold method to construct on-line peristimulus time histograms. More precise spike discrimination was performed off-line using a templatematching method. Eye position was monitored and recorded at $1 \mathrm{kHz}$ using the scleral search coil technique (Fuchs and Robinson 1966; Judge et al. 1980) (MEL-250UD; Enzanshi Kogyo, Tokyo).

Neurons were sought by advancing the recording electrode using an oil hydraulic micromanipulator (MO-95; Narishige, Tokyo) as the monkeys performed the visual search task during which the radius and orientation of the array were randomly varied from trial to trial. Once a neuron was isolated, we initially assessed the location of the receptive field by presenting a single stimulus oriented in six directions typically at $10^{\circ}$ eccentricity $\left(10.3 \pm 0.4^{\circ}\right.$, ranging from 9.2 to $11.7^{\circ}$ ) around the fixation spot in the fixation or memory-guided saccade task. If the neuron remained well isolated after the recording in the visual search task was finished, the angular extent of the receptive field was determined more precisely by presenting a single stimulus at the six locations where the six elements of the stimulus array were presented in the visual search task.

\section{Data analysis}

In this study, we focused on those neurons that conveyed signals related to target selection (i.e., their activity significantly discriminated the target from the distractor and nontarget stimuli). For this purpose, we performed a four-way ANOVA, with search dimension (shape search and color search), behavioral relevance (target, distractor and nontarget), shape (bar and circle), and color (cyan and yellow) as independent variables, to determine the dependence of neural activity on those task variables. In the ANOVA, effects were considered significant when the value of $P$ was $<0.001$. Further analysis was conducted with those neurons in which the effects of behavioral relevance were significant (the corresponding factors are indicated by "\#” symbols in Table 1). In addition, we computed eta-squared $\left(\eta^{2}\right)$, which is the proportion of the total variance that is attributable to an effect. $\eta^{2}$ is defined as the ratio of the effect variance $\left(\mathrm{SS}_{\text {effect }}\right)$ to the total variance $\left(\mathrm{SS}_{\text {total }}\right): \eta^{2}=\mathrm{SS}_{\text {effect }} / \mathrm{SS}_{\text {total }}$, as an estimate of effect size. Eta-squared indicates how much of the total variance in the data can be accounted for by a main effect or an interaction effect of independent variables. Only correct trials were analyzed and a squareroot transformation of firing rates was performed before the ANOVA to convert neural activity with a Poisson distribution to a normal distribution. Unless otherwise indicated, we analyzed neural activity occurring during a period extending from 70 to $220 \mathrm{~ms}$ after stimulus onset: this corresponds to the shortest visual latency in the PPC (Barash et al. 1991a; but see Bisley et al. 2004) and is 20-50 ms shorter than the average latency to the onset of the saccades (monkey Q, $241 \mathrm{~ms}$; monkey L, $273 \mathrm{~ms}$ ).

Saccades were detected using a computer algorithm that identified the initiation and termination of each saccade using velocity threshold criteria. Eye velocity was calculated by digitally differentiating the eye position signal. The initiation of a saccade was detected as the time at which the eye movement velocity exceeded $30 \% \mathrm{~s}$ for $\geq 5 \mathrm{~ms}$ and termination was detected as the return of the velocity to a level below that value. Saccade latency in the visual search task was measured as the interval from the appearance of the stimulus array to the beginning of the saccade.

Manifestation of delay-period and saccade-burst activity was examined in the memory-guided saccade task (Hikosaka and Wurtz 1983). For statistical analyses, activity was measured as the spike count per trial in three different trial intervals: the last $200 \mathrm{~ms}$ of the fixation period before target presentation (baseline activity); a 200-ms interval in the delay period, starting $350 \mathrm{~ms}$ before the fixation spot disappeared (delay-period activity); and a 200-ms interval starting 100 $\mathrm{ms}$ before initiation of a saccade and ending $100 \mathrm{~ms}$ after its initiation (saccade-burst activity). A neuron was defined as exhibiting delay-

TABLE 1. Numbers of neurons showing significant dependence on the task variables when tested by a four-way ANOVA

\begin{tabular}{|c|c|c|c|c|c|c|}
\hline \multirow[b]{3}{*}{ Factor } & \multicolumn{6}{|c|}{ Neuron Type } \\
\hline & \multicolumn{2}{|c|}{ Variant $(n=23)$} & \multicolumn{2}{|c|}{ Feature $(n=11)$} & \multicolumn{2}{|c|}{ Invariant $(n=35)$} \\
\hline & $N$ & Effect Size, \% & $N$ & Effect Size, \% & $N$ & Effect Size, \% \\
\hline Dim & 9 & $2.7 \pm 3.4$ & 1 & $0.8 \pm 0.9$ & 6 & $1.8 \pm 2.7$ \\
\hline Target\# & 13 & $6.2 \pm 4.5^{*}$ & 11 & $24.4 \pm 14.8 *$ & 35 & $32.5 \pm 19.6^{*}$ \\
\hline Shape & 9 & $8.6 \pm 13.1^{*}$ & 6 & $6.7 \pm 7.2 *$ & 10 & $1.5 \pm 2.2$ \\
\hline Color & 1 & $0.4 \pm 0.7$ & 5 & $10.7 \pm 16.6^{*}$ & 3 & $0.7 \pm 1.0$ \\
\hline Dim $\times$ Target\# & 7 & $3.4 \pm 3.5$ & 0 & $0.4 \pm 0.3$ & 2 & $0.9 \pm 0.9$ \\
\hline Dim $\times$ Shape & 1 & $0.3 \pm 0.3$ & 0 & $0.2 \pm 0.4$ & 0 & $0.4 \pm 0.7$ \\
\hline Dim $\times$ Color & 0 & $0.2 \pm 0.2$ & 0 & $0.2 \pm 0.3$ & 0 & $0.2 \pm 0.3$ \\
\hline Target $\times$ Shape\# & 15 & $7.3 \pm 5.7 *$ & 1 & $2.1 \pm 2.9$ & 1 & $0.6 \pm 0.6$ \\
\hline Target $\times$ Color\# & 0 & $0.7 \pm 0.7$ & 3 & $1.8 \pm 2.5$ & 0 & $0.7 \pm 0.7$ \\
\hline Shape $\times$ Color & 0 & $0.2 \pm 0.3$ & 2 & $0.7 \pm 1.0$ & 1 & $0.4 \pm 0.6$ \\
\hline Dim $\times$ Target $\times$ Shape\# & 14 & $9.9 \pm 11.1^{*}$ & 1 & $0.8 \pm 0.9$ & 1 & $0.7 \pm 0.8$ \\
\hline Dim $\times$ Target $\times$ Color $\#$ & 0 & $0.4 \pm 0.3$ & 1 & $0.8 \pm 0.5$ & 0 & $0.5 \pm 0.6$ \\
\hline Dim $\times$ Shape $\times$ Color & 0 & $0.2 \pm 0.2$ & 0 & $0.2 \pm 0.4$ & 0 & $0.4 \pm 0.5$ \\
\hline Target $\times$ Shape $\times$ Color $\#$ & 0 & $0.3 \pm 0.4$ & 1 & $0.9 \pm 1.0$ & 1 & $0.7 \pm 0.6$ \\
\hline Dim $\times$ Target $\times$ Shape $\times$ Color $\#$ & 0 & $0.4 \pm 0.5$ & 0 & $0.2 \pm 0.1$ & 0 & $0.4 \pm 0.3$ \\
\hline
\end{tabular}

Values of effect size are means \pm SD averaged across the neurons in each neuron group. Numbers of neurons showing significant dependence on the task variables and the effect size were evaluated using a four-way ANOVA in which search dimension (Dim), behavioral relevance (Target), shape (Shape), and color (Color) served as independent variables. Results of the ANOVA were separately described for the three neuron types: variant type, feature type, and invariant type. $N$ indicates the number of neurons that showed a significant dependence on the task variables. In the ANOVA, values of $P<0.001$ were considered significant. Superior symbols (\#) indicate the main and interaction effects in which the behavioral relevance factor (Target) was involved. Asterisks ( $*$ ) indicate the conditions under which the mean values of the effect size were $>5 \%$. 
period activity if activity during the delay-period was significantly greater than the baseline activity (Mann-Whitney $U$ test, $P<0.01$ ). A neuron was defined as exhibiting saccade-burst activity if activity during the saccade period was significantly greater than the preceding delay-period activity.

To quantify the angular extent of the receptive field for each neuron, the discharge rate measured at the six positions in the fixation or memory-guided saccade tasks was fit with a cubic spline interpolation function (MATLAB, The MathWorks, Natick, MA). For each neuron, the width of the directional-tuning curve was evaluated by computing its width at half-height from the background activity to the peak activity (Fig. 2). In the calculation of average population responses, the discharge rates at the six positions were divided by the peak activity for each neuron so that the peak activity was 1 . Then, the responses of each neuron were aligned so that the target location stimulating the most activity was located at $0^{\circ}$, fitted with a spline curve, and averaged across the neurons.

To compare neural activities across the population of recorded neurons, the normalized responses (Figs. $3 E, 4 E$, and 6) were calculated by dividing the response obtained under each trial condition by the response averaged across the entire 24 trial conditions (two shapes $\times$ two colors $\times$ three behavioral relevance $\times$ two search dimensions). So, the grand average of the normalized responses across the total 24 conditions was equal to 1 . One advantage of this normalization method was that the total power of the responses for individual neurons was maintained at the same value, even when the response selectivity largely differed from neuron to neuron.

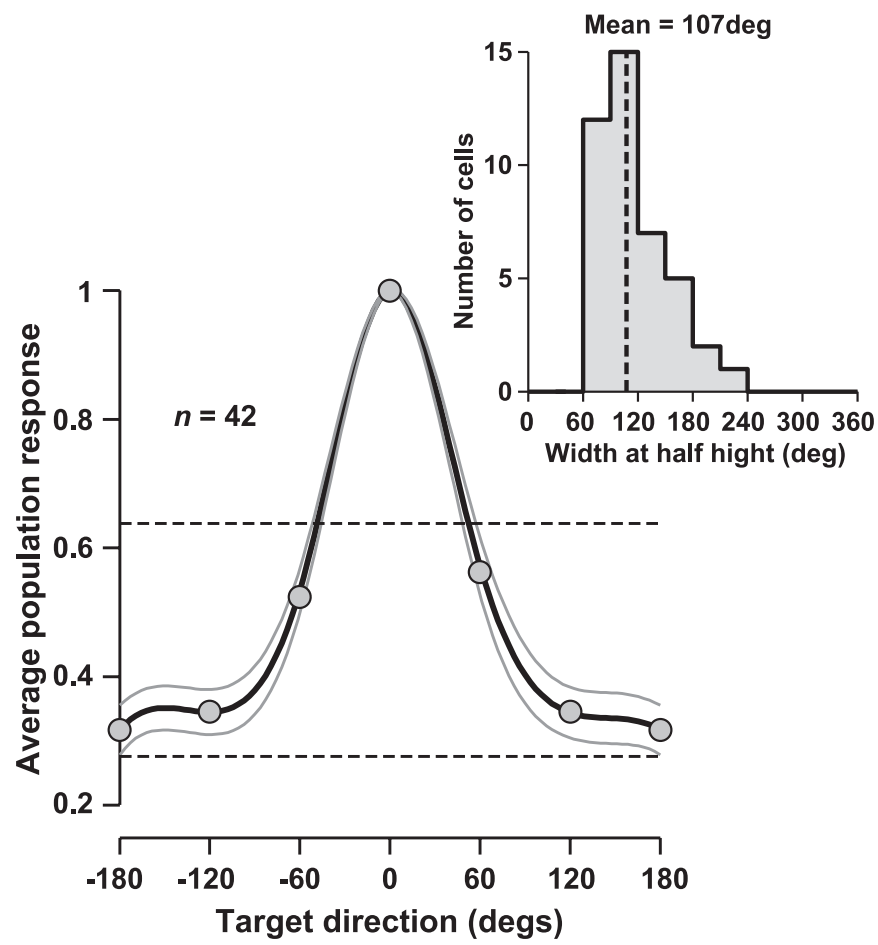

FIG. 2. Angular extent of the receptive field. The angular extent of the receptive field was evaluated using the responses of 42 neurons (mean and SE). The responses to a single stimulus at the 6 locations around the fixation spot were aligned for each cell so that the target location eliciting the most activity was aligned at $0^{\circ}$. The responses were then fitted with a cubic spline interpolation function and averaged across neurons. Positive angles progress counterclockwise. The bottom horizontal dashed line represents the average background activity measured during the fixation period for all target directions; the top one represents the average half-height from the background activity to the peak activity. The points at $+180^{\circ}$ and $-180^{\circ}$ were calculated from the same data set. Top-right inset: the histogram shows the distribution of widths at the half-height for each neuron.
Population responses (Fig. 7) were calculated by averaging the spike density functions of individual neurons, which were constructed by convolving spike trains with a Gaussian function $(\mathrm{SD}=10 \mathrm{~ms}$ ) (Richmond et al. 1987). Significant differences between the population responses elicited by the target and those elicited by the other stimuli were determined using the permutation test (Efron and Tibshirani 1993). In each permutation, trial data for each neuron were randomly shuffled across the responses to two different stimuli and were divided into two groups (the number of trials in each group was the same as for the actual data). Permuted spike density functions for each neuron were calculated in the same way as for the actual data, after which the permuted population responses were calculated by averaging the spike density functions across the neurons. This procedure was iterated to produce 1,000 total permutations. If the difference between the actual population responses elicited by the target and other stimuli was larger than the permuted differences in $>990$ iterations, it was deemed that the difference was significant (at the $P<0.01$ level). We moved time points in 1 -ms increments after stimulus presentation and tested whether the population response to the target was significantly larger than that to the distractor and nontarget.

To quantify the dependence on the shape or color features (Fig. 9), shape and color preference indices were calculated as

$$
\begin{aligned}
& \text { Shape index }=\left|\mathrm{R}_{11}+\mathrm{R}_{12}-\mathrm{R}_{21}-\mathrm{R}_{22}\right| /\left(\mathrm{R}_{11}+\mathrm{R}_{12}+\mathrm{R}_{21}+\mathrm{R}_{22}\right) \\
& \text { Color index }=\left|\mathrm{R}_{11}-\mathrm{R}_{12}+\mathrm{R}_{21}-\mathrm{R}_{22}\right| /\left(\mathrm{R}_{11}+\mathrm{R}_{12}+\mathrm{R}_{21}+\mathrm{R}_{22}\right)
\end{aligned}
$$

where $R_{i j}(i=1,2 ; j=1,2)$ indicates the magnitude of the response when the stimulus features of the target appearing in the receptive field were the ith shape (first shape $=$ bar; second shape $=$ circle) and the $\mathrm{jth}$ color (first color = cyan; second color $=$ yellow). Since dependence on the shape or color feature was markedly different under the two search conditions, the indices were calculated separately for each of the shape and color search conditions.

\section{Recording site}

Neuronal activities were recorded from the lateral bank of the intraparietal sulcus in both monkeys. Using a set of grids, neuronal activity was explored through microelectrode penetrations spaced at approximately $0.5-\mathrm{mm}$ intervals in the rostrocaudal direction along the intraparietal sulcus. The location of the sulcus was specified based on the response properties: the superior parietal gyrus (area 5) exhibits activity related to somatosensory stimuli, whereas the inferior parietal gyrus exhibits visual and saccade-related responses (Barash et al. 1991a; Mountcastle et al. 1975). We did not record from the inferior parietal gyrus. Recording sites were identified based on their depth below the dura, the delay-period activity in the memory-guided saccade task, and X-ray localization of the recording electrode on structural MR images obtained prior to the start of recording. Given that we recorded from the lateral bank of the intraparietal sulcus and that many sites exhibited significant delay-period activity, we think that we mainly recorded from the LIP. However, a small proportion of the neurons $(10 / 73,14 \%)$ recorded at sites within the first $2.5 \mathrm{~mm}$ of penetration might have belonged to area 7a (Barash et al. 1991a; Ben Hamed et al. 2002). For this reason, we will use the term "PPC" to indicate the region from which we recorded.

We discriminated the anterior portion of the lateral bank of the intraparietal sulcus (AIP) from the LIP. In the AIP, immediately rostral to the LIP, three-dimensional shape-selective and hand-manipulation-related activities were recorded (Murata et al. 2000; Sakata and Taira 1994; Sakata et al. 1995). In previous studies of the AIP, the caudal border of this area was identified by the presence of visual fixation or saccade-related neurons and by the absence of handmanipulation-related neurons (Murata et al. 2000). The LIP was therefore distinguished from the AIP by the presence of large numbers 
of neurons with strong saccade-related activity. In the present study, we recorded from a region where strong delay-period and saccadeburst activities were observed during the memory-guided saccade task. The neurons studied here were unlikely those in the AIP.

Positions of the recording electrodes in the PPC were regularly checked by X-ray during the experiments. For further confirmation, the recording sites in one of the monkeys were histologically reconstructed (Fig. 10). In the last few recording sessions of monkey Q, the position of the recording electrode was marked by making three electrolytic microlesions by passing a small DC current $(5 \mathrm{~mA}, 120 \mathrm{~s})$ through the recording electrode at different sites and depths. After the recordings were complete, the monkey was deeply anesthetized with sodium pentobarbital and then perfused through the heart with saline containing $3.6 \%$ formalin fixative and $10 \%$ sucrose. The brain was excised, sliced into 50- $\mu \mathrm{m}$ sections in the coronal plane, and stained with cresyl violet. The sections were examined under a microscope and the sites of the microlesions were identified. Histological reconstruction matched the locations determined using MR images and X-ray photographs.

\section{R E S U L T S}

\section{Behavioral performance}

The monkeys' behavioral performances during the visual search task were well above the chance level $(1 / 6=16.7 \%)$. The average performance scores under the shape and color search conditions were, respectively, 82.8 and $85.6 \%$ for monkey Q and 87.0 and $91.0 \%$ for monkey L during the recordings sessions. The average saccade reaction times (with $\mathrm{SD}$ ) in the shape and color searches were, respectively, $243 \pm 34$ and $238 \pm 38 \mathrm{~ms}$ for monkey $\mathrm{Q}$ and $273 \pm 49$ and $274 \pm 53 \mathrm{~ms}$ for monkey $\mathrm{L}$ during the recording sessions. There was no significant difference in either the performance or saccade reaction time between the two search conditions in either monkey (Mann-Whitney $U$ test, $P>0.1$ ), which suggests that the task difficulties were nearly the same for each monkey under the two search conditions.

\section{Neuronal database}

We recorded from 123 visually responsive single neurons in the PPC of the two monkeys. For 110 of those neurons, the data sets were large enough ( $>12$ repetitions of each trial condition) to enable analysis. Of those 110 neurons, 75 exhibited activity in which the effects of behavioral relevance were significant ( $P<0.001$, four-way ANOVA; see METHODS); however, two neurons were excluded because the location at which the largest activity was elicited when a single stimulus was used differed by $>120^{\circ}$ from the location at which the largest activity was elicited when using a target embedded within an array. The remaining 73 neurons were subjected to further analysis ( $n=30$, monkey Q; $n=43$, monkey L).

We were able to determine the directional-tuning curve for 42 of the 73 neurons studied (see METHODS). The angular extent of the receptive field was well localized around the direction eliciting the strongest response (Fig. 2). The mean value of the width at half-height was $107^{\circ}$ (Fig. 2, inset), which is comparable to that reported in a previous study of the LIP (Barash et al. 1991b).
Activity of posterior parietal neurons in the multidimensional visual search task

A majority of posterior parietal neurons showed increased activity when a monkey made a saccade to the target stimulus that appeared in its receptive field. In a substantial subset of neurons, however, such response modulation was strongly dependent on the stimulus features of the target and the target-defining dimension. Figure 3 shows one representative neuron. This neuron had a well-localized receptive field (width at halfheight $=82^{\circ}$ ) and exhibited no activity during the delay and saccade periods in the memory-guided saccade task (Mann-Whitney $U$ test, $P>0.05$ ), although other neurons recorded at shallower sites on the same electrode track exhibited strong delay-period activity. During the visual search task, the response patterns elicited in this cell by the target stimulus varied dramatically, depending on the stimulus features and the target-defining dimension. This neuron showed the greatest activity when the target was a yellow bar and the target-defining dimension was the shape dimension (Fig. 3A, red trace). Under this condition, the activity significantly discriminated the target from the distractor and nontarget stimuli (Mann-Whitney $U$ test, $P<0.001$ ). By contrast, when the stimulus features of the target were changed from a yellow bar to a cyan circle (Fig. 3B, red trace), when the target-defining dimension was changed from the shape dimension to the color dimension (Fig. 3C, green trace) or when both the stimulus features and the target-defining dimension were changed (Fig. 3D, green trace), the magnitude of the activity elicited by the target stimuli was significantly less than the maximal response (Mann-Whitney $U$ test, $P<$ 0.001 ) and was no longer sufficient to significantly discriminate the target (Mann-Whitney $U$ test, $P>0.05$ ).

Figure $3 E$ shows the normalized responses for the entire set of 24 trial conditions (two shapes $\times$ two colors $\times$ three behavioral relevance $x$ two search dimensions). These normalized responses were obtained by dividing the response for each trial condition by the response averaged across the entire 24 trial conditions. This neuron exhibited significant target discrimination only when the target was a bar stimulus and was defined in the shape dimension (Mann-Whitney $U$ test, $P<$ $0.001)$; significant target discrimination was not seen when the target stimulus was a circle or the target-defining dimension was the color dimension (Mann-Whitney $U$ test, $P>0.01$ ). Thus the activity related to target discrimination was highly selective for the combination of the stimulus features and the target-defining dimension. This was further confirmed by a four-way ANOVA in which search dimension, behavioral relevance, shape, and color served as independent variables (see METHODS). In addition to significant main effects of search dimension $\left[F_{(1,264)}=49.6, P<0.001, \eta^{2}=11.1 \%\right]$ and behavioral relevance $\left[F_{(2,264)}=25.3, P<0.001, \eta^{2}=11.4 \%\right]$, we found a significant interaction effect for search dimension, behavioral relevance, and shape $\left[F_{(2,264)}=15.3, P<0.001, \eta^{2}=\right.$ $6.9 \%]$ and for search dimension and shape $\left[F_{(1,264)}=22.0\right.$, $P<0.001, \eta^{2}=9.9 \%$ ]. The significant interaction of search dimension, behavioral relevance, and shape is consistent with the aforementioned view that increases in activity elicited by the target were highly selective for a particular combination of task variables.

Figure 4 shows another representative neuron whose response profile is very different from that of neuron in Fig. 3, in 


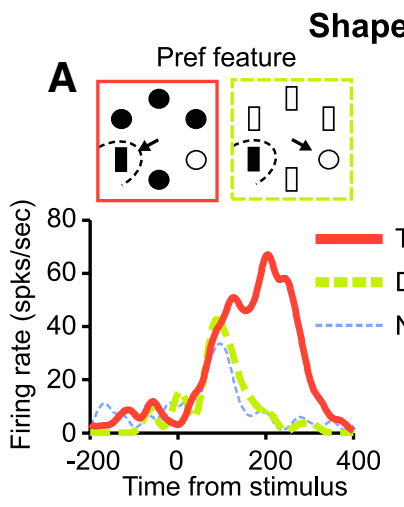

(ms)

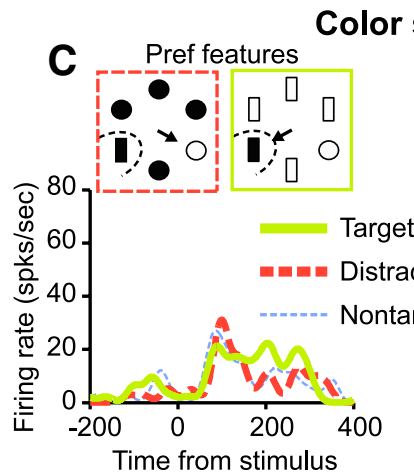

(ms)

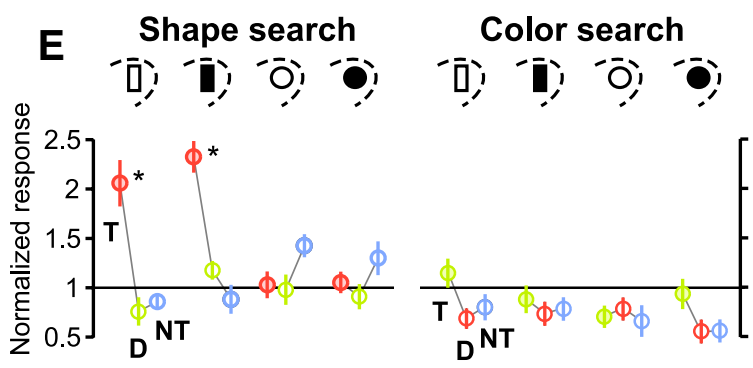

Shape singleton Color singleton $\bigcirc$ Nonsingleton

FIG. 3. A parietal neuron showing dependence on stimulus features and the target-defining dimension. $A-D$ : spike density functions during the multidimensional visual search task. Thick red and green lines indicate the responses to the shape and color singleton stimuli appearing in the receptive field. Dotted thin blue lines indicate the responses to a nontarget (nonsingleton) stimulus. Solid and dashed lines, respectively, indicate that the stimulus in the receptive field was the target or distractor. The responses are temporally aligned at the onset of the stimulus array. The spike density functions were smoothed with a Gaussian function $(\mathrm{SD}=10 \mathrm{~ms})$. Top insets in each panel show the stimulus conditions under which the stimulus in the receptive field was the target or distractor. Formats of the rectangular frames in the insets are the same as in the spike density functions. This neuron only exhibited a marked response to the target when a specific combination of the stimulus feature (bar shape) and the target-defining dimension (shape dimension) was met $(A)$. E: normalized mean responses (with SE) of the same neuron for the entire set of 24 trial conditions, which were calculated by dividing the responses to the 24 different stimulus conditions by their average. Top insets indicate the stimulus features in the receptive field in the shape search (left) and the color search (right). Data for the same stimulus features are connected by lines under each search condition. In each set, 3 connected circles respectively indicate from left to right that the stimulus in the receptive field was the target (T), distractor (D), or nontarget (NT). Red, green, and blue circles represent the responses to the shape singleton, the color singleton, and the nonsingleton stimuli, respectively. Asterisks indicate significant difference from the 2 other responses in each set (Mann-Whitney $U$ test, $P<0.01$ ).
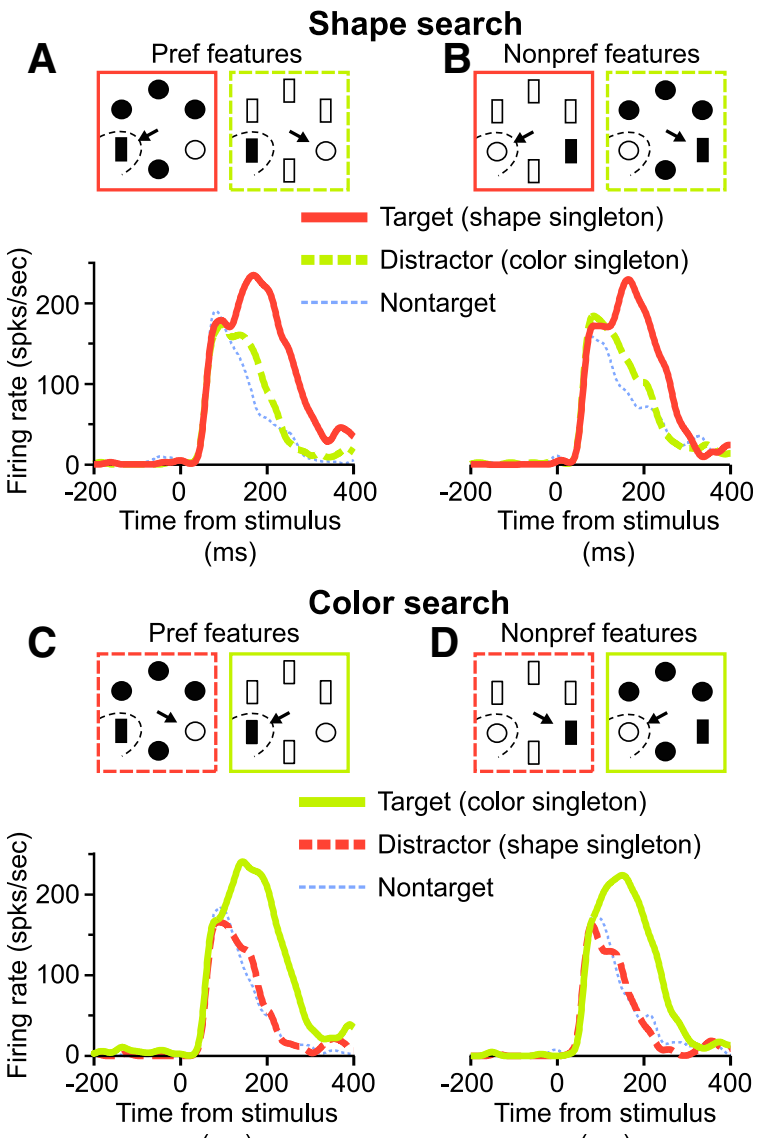

(ms)

(ms)

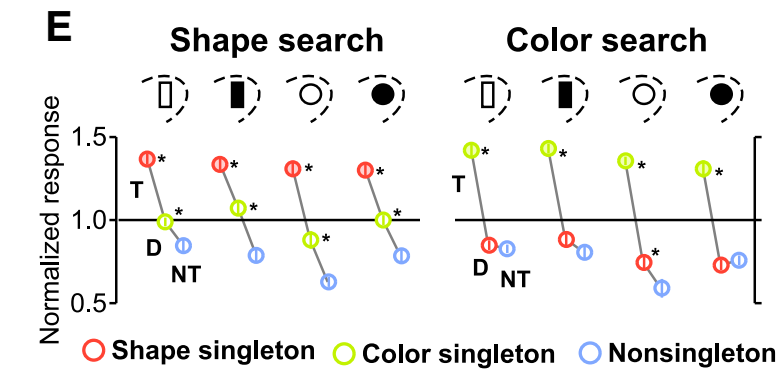

Color search

FIG. 4. A parietal neuron showing no dependence on stimulus features or the target-defining dimension. The activity of this neuron always exhibited significant discrimination of the target from the other stimuli, irrespective of the stimulus features and target-defining dimension. Conventions are the same as in Fig. 3.

that the activity elicited by the target was much less dependent on the stimulus features and the target-defining dimension during the visual search. This neuron exhibited significant delay-period and saccade-burst activities during the memoryguided saccade task (Mann-Whitney $U$ test, $P<0.01$ ). During the visual search task, the response magnitude was largest when the target was a yellow bar and the target-defining dimension was the color dimension (green trace in Fig. 4C) and the activity significantly discriminated the target from the distractor and nontarget stimuli (Mann-Whitney $U$ test, $P<$ $0.01)$. Notably, when the stimulus features of the target were changed to cyan and circle (Fig. $4 D$, green trace), when the target-defining dimension was changed to the shape dimension (Fig. 4A, red trace), or when both the stimulus features and the target-defining dimension were changed (Fig. 4B, red trace), 
the magnitude of the response to the target did not differ significantly from the maximal response (Mann-Whitney $U$ test, $P>0.1)$. Figure $4 E$ shows that under all eight target conditions the responses to the target were significantly larger than those to the distractor and nontarget stimuli (MannWhitney $U$ test, $P<0.001$ ), irrespective of the stimulus features and the target-defining dimension. The four-way ANOVA revealed that the effect of behavioral relevance was significant and the effect size was exceedingly large $\left[F_{(2,426)}=593.1\right.$, $\left.P<0.001, \eta^{2}=76.5 \%\right]$. Even though the effect of shape $\left[F_{(1,426)}=36.3, P<0.001, \eta^{2}=2.3 \%\right]$ and the interaction effect of search dimension and behavioral relevance $\left[F_{(2,426)}=\right.$ 18.3, $P<0.001, \eta^{2}=2.4 \%$ ] were also significant, their effect size were relatively small, compared with the effect of behavioral relevance.

\section{Population profile of activity}

As illustrated by the two examples summarized earlier, the activity related to target representation varied from neuron to neuron in the PPC. To quantify this variance in neuronal activity, we calculated two indices, "feature dependence" and "dimension dependence," using the three response patterns obtained under the various target conditions. First, as a reference condition, we chose from among the eight target conditions the one that elicited the greatest activity (e.g., Fig. 3A, red trace). Hereafter, the stimulus features and the target-defining dimension under this condition will be referred to as the preferred features and the preferred target-defining dimension. The complementary stimulus features and complementary target-defining dimension will be referred to as the nonpreferred stimulus features (e.g., if the preferred features were yellow and bar, the nonpreferred features would be cyan and circle) and the nonpreferred target-defining dimension (e.g., if the preferred target-defining dimension was the shape dimension, the nonpreferred target-defining dimension would be the color dimension). Second, we chose the target condition under which the stimulus features of the target were the nonpreferred ones, but the target-defining dimension remained the same as that under the reference condition (e.g., Fig. 3B, red trace). The feature dependence was then defined as the difference in response strength between the reference and this nonreference condition divided by the former. This index reflects dependence of the response elicited by the target on the stimulus features. Third, the dimension dependence was determined in a similar manner. In that case, we chose the target condition under which the target-defining dimension was the nonpreferred dimension, whereas the stimulus features of the target were kept the same as those under the reference condition (e.g., Fig. 3C, green trace). The dimension dependence was then defined as the difference in the response between the reference and this nonreference condition divided by the former. This index reflects the dependence of the response elicited by the target on the target-defining dimension. Notably, when the target-defining dimension was switched, not only the search dimension but also the singleton type of the target stimulus was altered. Indeed, we actually found a significant effect of singleton type on activity in a subset of PPC neurons. This will be examined in the following text (see Fig. 6, $A$ and $B$ ).

For each index, a value of 1 indicates that a significant response was observed only for a specific target with particular stimulus features and/or under a particular target-defining dimension, whereas a value of 0 indicates that there was no difference in the responses elicited by the target under reference and nonreference conditions. Thus the neuron with the response profile shown in Fig. 3 will have values near 1 for both indices, whereas that shown in Fig. 4 will have values near 0 for both indices.

Figure 5 shows the distribution of the values of feature and dimension dependence indices for the neurons analyzed in this study. The data points corresponding to the two example neurons (Figs. 3 and 4) are indicated by arrows. Overall, data points with weaker feature dependence were associated with weaker dimension dependence, whereas those with stronger feature dependence were associated with stronger dimension dependence. The correlation between the two indices was significant $(r=0.61, P<0.001)$ and the data points did not appear to form discrete clusters; instead, they were distributed continuously. Nonetheless, to examine the differences in response profiles at the population level, we divided the neurons into four types based on whether each of the indices was significantly different from 0 . For $23(32 \%)$ neurons, the values of both indices were significantly different from 0 (open symbols; permutation analysis, $P<0.01$ ), indicating that the responses to the target varied depending on the stimulus features and the target-defining dimension. These will be referred to as "variant

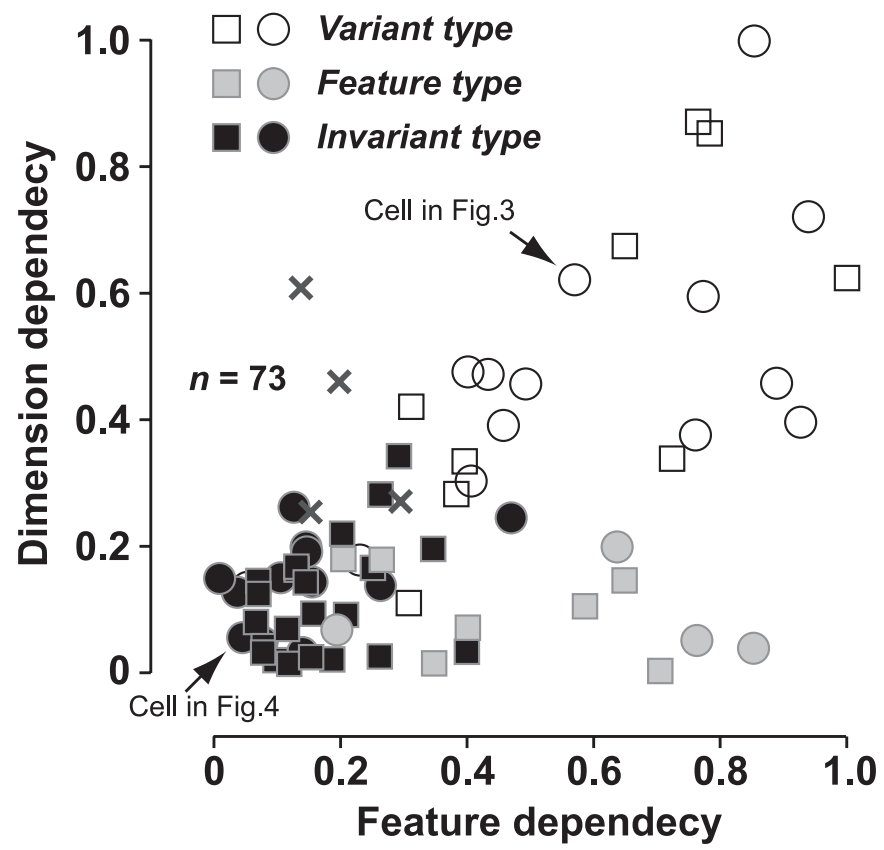

FIG. 5. Dependence of target discrimination on stimulus features and target-defining dimension. The distribution of feature dependence and dimension dependence indices of 73 neurons. Each symbol represents one neuron. A dimension dependence index with a value of 0 indicates that the strength of response to the target did not vary when the target-defining dimension was changed from the preferred dimension to the nonpreferred one, whereas a value of 1 indicates that the response to the target vanished when the target-defining dimension was changed to the nonpreferred one. Values of the feature dependence index are similar in meaning. Open symbols indicate that both indices are significantly different from 0 (permutation test, $P<0.01$ ); filled black symbols indicate that they are not (permutation test, $P>0.01$ ). Gray symbols indicate that the feature dependence index is significantly different from 0 (permutation test, $P<0.01$ ), but the dimension dependence index is not (permutation test, $P>0.01$ ). Open, filled, and gray symbols correspond to the variant-, feature-, and invariant-type neurons, respectively. Circles and squares indicate neurons from monkey $\mathrm{Q}$ and monkey L, respectively. 
type"; the first representative neuron (Fig. 3) was of this type. For $11(15 \%)$ neurons, feature dependence alone was significantly different from 0 (filled gray symbols), indicating that the responses to the target varied depending only on stimulus features. These will be referred to as "feature type." For 35 $(48 \%)$ neurons, neither index was significantly different from 0 (filled black symbols; permutation analysis, $P>0.01$ ), indicating that the responses to the target were invariant with respect to the stimulus features and target-defining dimension. These will be referred to as "invariant type"; the second example neuron (Fig. 4) was of this type. Finally, for the remaining 4 neurons, dimension dependence alone was significantly different from 0 (cross symbols). Because of their small number, we did not make further analysis of this type of neuron.

In Fig. 5, we considered only the responses elicited when the target stimulus appeared in the receptive field. To know the properties of the responses when the distractor or nontarget stimulus appeared in the receptive field, we calculated the normalized responses (as shown in Figs. $3 E$ and $4 E$ ) for the three neuron types defined in Fig. 5. Figure 6 shows the normalized responses to the target, distractor, and nontarget stimuli (T, D, NT) when these stimuli had the preferred or nonpreferred stimulus features (PF, NF) and appeared in the receptive field in the shape search (left column) or the color search (right column). Figure 6, $A$ and $B$ shows the normalized responses of the variant-type neurons $(n=23)$. For clarity, the variant-type neurons were further classified into two groups according to whether the preferred target-defining dimension was the shape dimension or the color dimension. Figure $6 \mathrm{~A}$ shows the normalized responses of the 21 variant-type neurons that preferred the target defined in the shape dimension. Thin gray lines indicate the responses of individual neurons and thick black lines indicate their population average. Under the shape search condition, a target with the preferred features (PFs) elicited an increase in activity that significantly discriminated the target from the other stimuli (Mann-Whitney $U$ test, $P<0.01$ ). However, when the target had the nonpreferred features (NFs), the significant target discrimination was lost (NF, MannWhitney $U$ test, $P>0.1$ ). Under the color search condition, the target elicited no substantial increase in activity and there was no significant target discrimination (Mann-Whitney $U$ test, $P>0.05$ ). Figure $6 B$ shows the normalized responses of the two variant-type neurons that exhibited the greatest activity when the target was defined in the color dimension. Because of the small cell number, the average responses were not calculated in this case, although the same tendency was observed in the response profiles: the target elicited a substantial increase in activity only when it had the preferred features and was defined in the preferred (i.e., color) dimension. Thus in the variant-type neurons, the increase in activity elicited by the target was highly selective for the combination of the stimulus features and the target-defining dimension.

Previous studies have shown that PPC neurons automatically detect and encode the location of salient singleton stimuli, even when they are not behaviorally relevant (Constantinidis and Steinmetz 2005). We found a similar effect of singleton type on the activity of some variant-type neurons, irrespective of the behavioral relevance of the singleton stimulus. With our experimental design, a shape singleton served as the distractor in the color search, whereas a color singleton served as the distractor
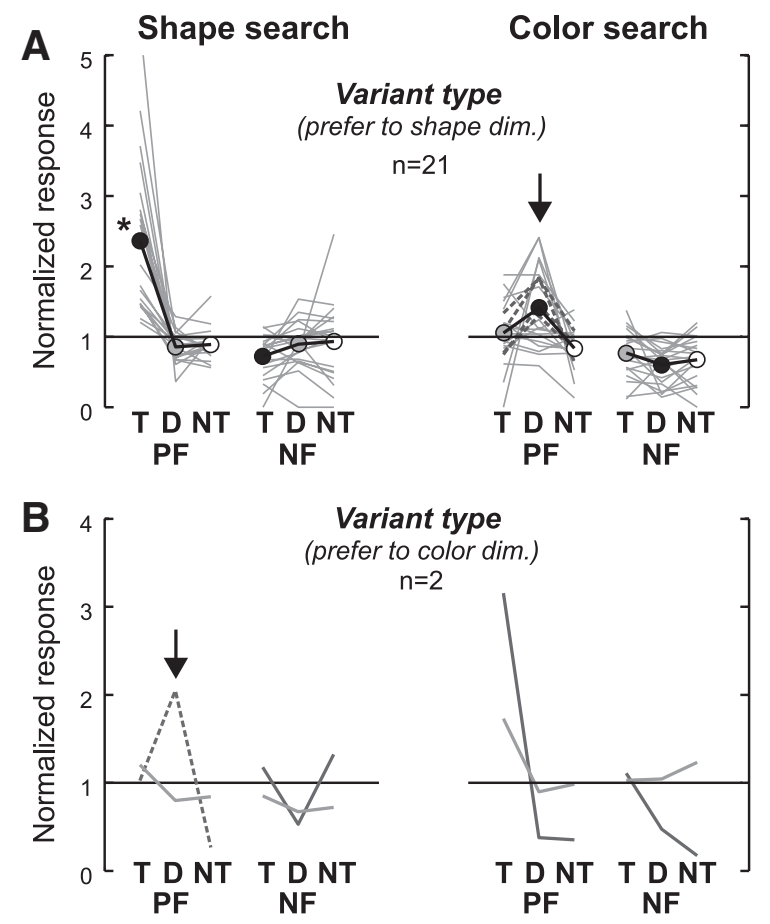

Variant type
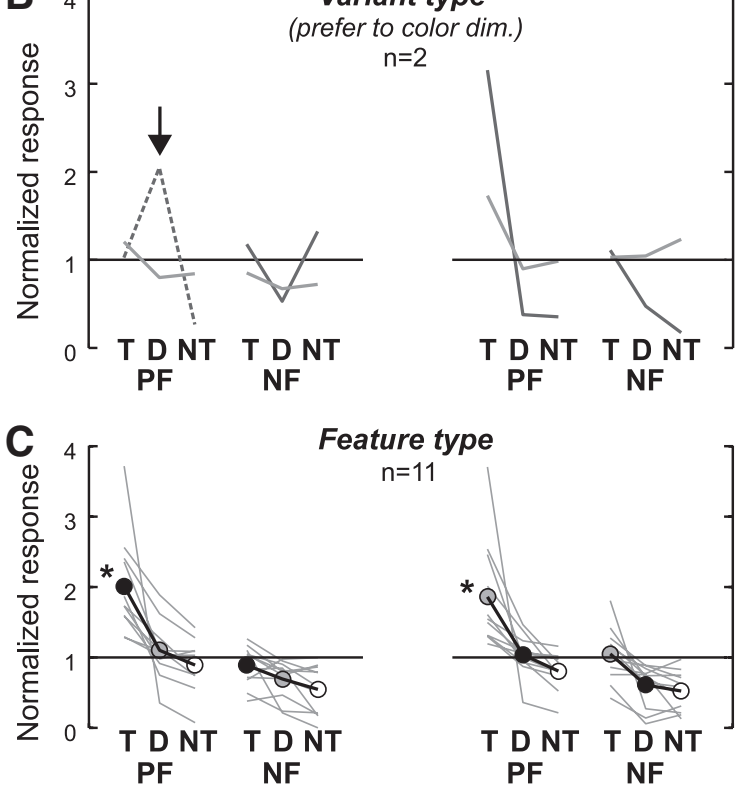

Feature type $n=11$
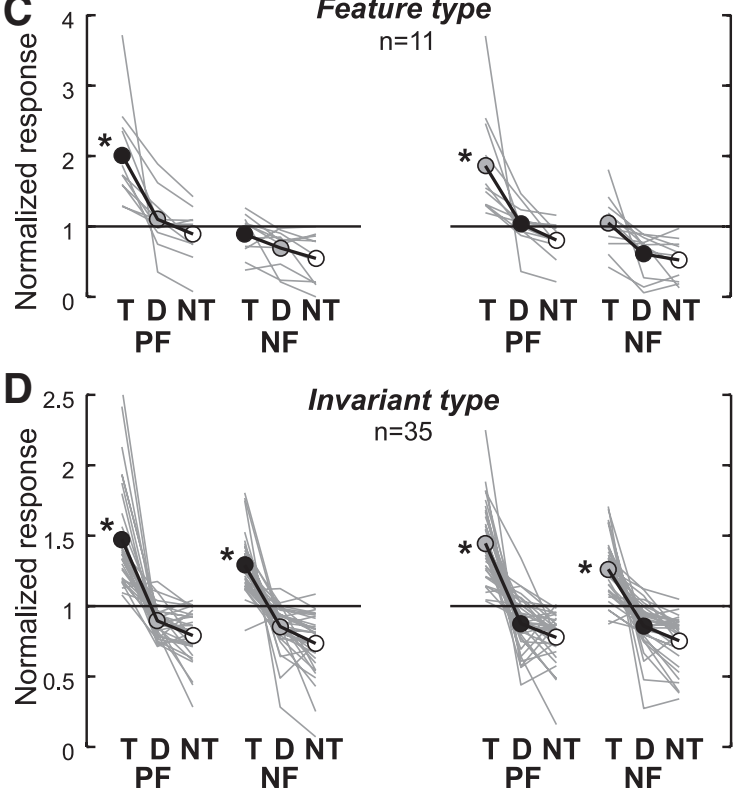

Invariant type

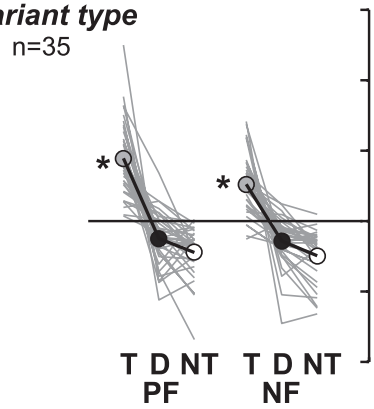

Shape singleton $\bigcirc$ Color singleton $\bigcirc$ Nonsingleton

FIG. 6. Normalized population responses. $A$ and $B$ : normalized responses of the variant-type neurons $(n=23)$. For clarity, these neurons were separated into the 2 groups based on whether the preferred target-defining dimension was shape $(A, n=21)$ or color $(B, n=2)$. The normalized responses for each neuron were calculated by dividing the responses to each of the 24 different stimulus conditions by their average. Of the 24 responses, 12 are shown: the responses are to the target, distractor, and nontarget stimuli (T, D, NT) when they appeared in the receptive field with the preferred or nonpreferred stimulus features $(\mathrm{PF}, \mathrm{NF})$ under the shape (left column) or color (right column) search conditions. Thin gray lines indicate the responses of individual neurons and thick black lines indicate their population average. Data for the same stimulus features in the receptive field are connected under each of the search conditions. From left to right, the 3 circles in each set correspond to the target (T), distractor (D), and nontarget (NT) conditions. The asterisk indicates a significant difference from the 2 other responses in each set. $C$ : normalized responses of the feature-type neurons $(n=11)$. $D$ : normalized responses of the invariant-type neurons $(n=35)$. 
in the shape search (arrow in Fig. 6, $A$ and $B$ ). In 11 of the 21 neurons that preferred the shape dimension, the response to the distractor singleton was greater than the responses to the target and nontarget, although this difference was significant for only 4 neurons (dashed lines in Fig. 6A; Mann-Whitney $U$ test, $P<$ 0.01 ) and was not significant for the population average (MannWhitney $U$ test, $P>0.05)$. A similar significant effect of the singleton type was also found in one of the two variant neurons that preferred the color dimension (a dashed line in Fig. $6 B$; Mann-Whitney $U$ test, $P<0.01$ ). These findings demonstrate that a subset of PPC neurons were able to specify the locus of the salient stimulus defined by one specific feature dimension rather than the locus of the behaviorally relevant target.

Figure $6 C$ shows responses of the 11 feature-type neurons. When the target in the receptive field had the preferred stimulus features, these neurons exhibited enhanced activity that significantly discriminated the target from other stimuli (MannWhitney $U$ test, $P<0.01$ ), irrespective of the ongoing search dimension. The activity of this type of neuron was thus profoundly dependent on the stimulus features of the target, irrespective of the target-defining dimension. Figure $6 D$ shows the normalized responses of the 35 invariant-type neurons. These neurons always responded significantly more strongly when the target appeared in their receptive field than when the other stimuli appeared (Mann-Whitney $U$ test, $P<0.01$ ). As a result, this type of neuron significantly discriminated the target from the other stimuli, irrespective of the stimulus features or the target-defining dimension.

To determine the differences in the degree of association between the task variables and their effect on activity among the three types of neurons (variant, feature, and invariant types), we conducted a four-way ANOVA for each of the neuron types (Table 1). Among the variant-type neurons, the effect of the interaction of search dimension, behavioral relevance, and shape (Dim $\times$ Target $\times$ Shape) and that of behavioral relevance and shape (Target $\times$ Shape) were significant in more than half of the neurons ( $n=14,67 \%$ and $n=$ $15,71 \%$, respectively) and the effect sizes accounted for substantial amounts of the total variation in activity $\left(\eta^{2}=9.9\right.$ and $7.3 \%$, respectively). This confirms our view that the target elicited a significant increase in activity only when specific stimulus features and a particular target-defining dimension were combined.

Among the feature-type neurons, by contrast, none of the interaction effects was significant in more than one third of the neurons. Instead, the main effects of shape (Shape) and color (Color) were significant in about half of the neurons $(n=6$, $55 \% ; n=5,45 \%$, respectively) and the size of the effect was substantial $\left(\eta^{2}=6.7\right.$ and $10.7 \%$, respectively), indicating that the effects of stimulus features are crucial for the activity of feature-type neurons. Further, the main effect of behavioral relevance (Target) was significant in all the feature-type neurons $\left(n=11,100 \% ; \eta^{2}=24.4 \%\right)$. This was caused by the fact that the activity for the target stimulus tended to be larger than that for the other stimuli, even when the stimulus features of the target were not the preferred ones (Fig. 6C).

In the invariant-type neurons, the main effect of behavioral relevance (Target) was significant in all the neurons $(n=35$, $100 \%)$ and the effect size was large $\left(\eta^{2}=32.5 \%\right)$, compared with the effect sizes of the other factors $\left(\eta^{2}<2 \%\right)$. This means that the effect of the behavioral relevance of the receptive field stimulus was predominant in the activity of the invariant-type neurons. Thus results of the four-way ANOVA analysis highlight the differences in the response profiles among the variant-, feature-, and invariant-type neurons and confirm the results depicted in Fig. 6.

\section{Time course of neural representation of target information}

To determine whether the increases in activity elicited by the targets were transient phenomena, occurring only after array presentation, or were sustained until saccade initiation, we examined the time course of the average population responses elicited by the target, distractor, and nontarget stimuli. Population responses were calculated by averaging the spike density functions of the individual neurons. The significance of differences between the population responses elicited by the target and those elicited by other stimuli was determined using permutation tests (see METHODS).

Figure 7, $A-D$ shows activity averaged across the varianttype neurons. For clarity, only the neurons that preferred the shape dimension were considered $(n=21)$. The response was significantly greater when the target stimulus in the receptive field had the preferred stimulus features and was defined in the shape dimension (Fig. 7A, red solid trace) than when the identical stimulus was the distractor or the nontarget (Fig. 7A, green dashed and blue dotted thin traces). The differences between the responses elicited by the target and those elicited by the distractor or nontarget stimuli became significant 115 ms after array presentation and remained significant until saccade initiation (horizontal black line, permutation test, $P<$ 0.01). However, when the stimulus features of the target were changed to the nonpreferred ones (Fig. $7 B$, red trace) or when the target was defined in the color dimension (Fig. 7, $C$ and $D$, green traces), there was no evident increase in the response to the target and the significant target discrimination disappeared (permutation test, $P>0.01$ ).

Previous studies have demonstrated that the activity of LIP neurons varied with the latency of saccade (Ipata et al. 2006b; Thomas and Paré 2007). This raises the possibility that the selectivity of the variant-type neurons may change depending on the latency of impeding saccade. To assess this issue, we divided the trials into two equally sized groups according to the latency of saccade (average latencies were 237 and $271 \mathrm{~ms}$ ) and examined whether the response selectivity for the 24 trial conditions (two shapes $\times$ two colors $\times$ three behavioral relevance $X$ two search dimensions) was modified depending on the saccade latency. The response selectivity was well maintained between the two saccade latency groups (correlation coefficient, $r=0.96 ; P<0.001)$. Further, a two-way ANOVA analysis in which saccade latency and trial condition served as independent variables revealed that there was no significant effect of saccade latency $\left[F_{(1,23)}=3.25, P>0.05\right]$. These results suggest that the response selectivity in the variant neurons was largely independent of the latency of saccade.

Figure 7, $E-H$ shows the activity averaged across the feature-type neurons $(n=11)$. When the target had the preferred features in the shape search (Fig. $7 E$, red trace) or in the color search (Fig. 7G, green trace), the activity significantly discriminated the target from the other stimuli, beginning around 140-160 ms after array presentation, and this significant effect lasted until saccade initiation (permutation test, $P<0.01$ ). 

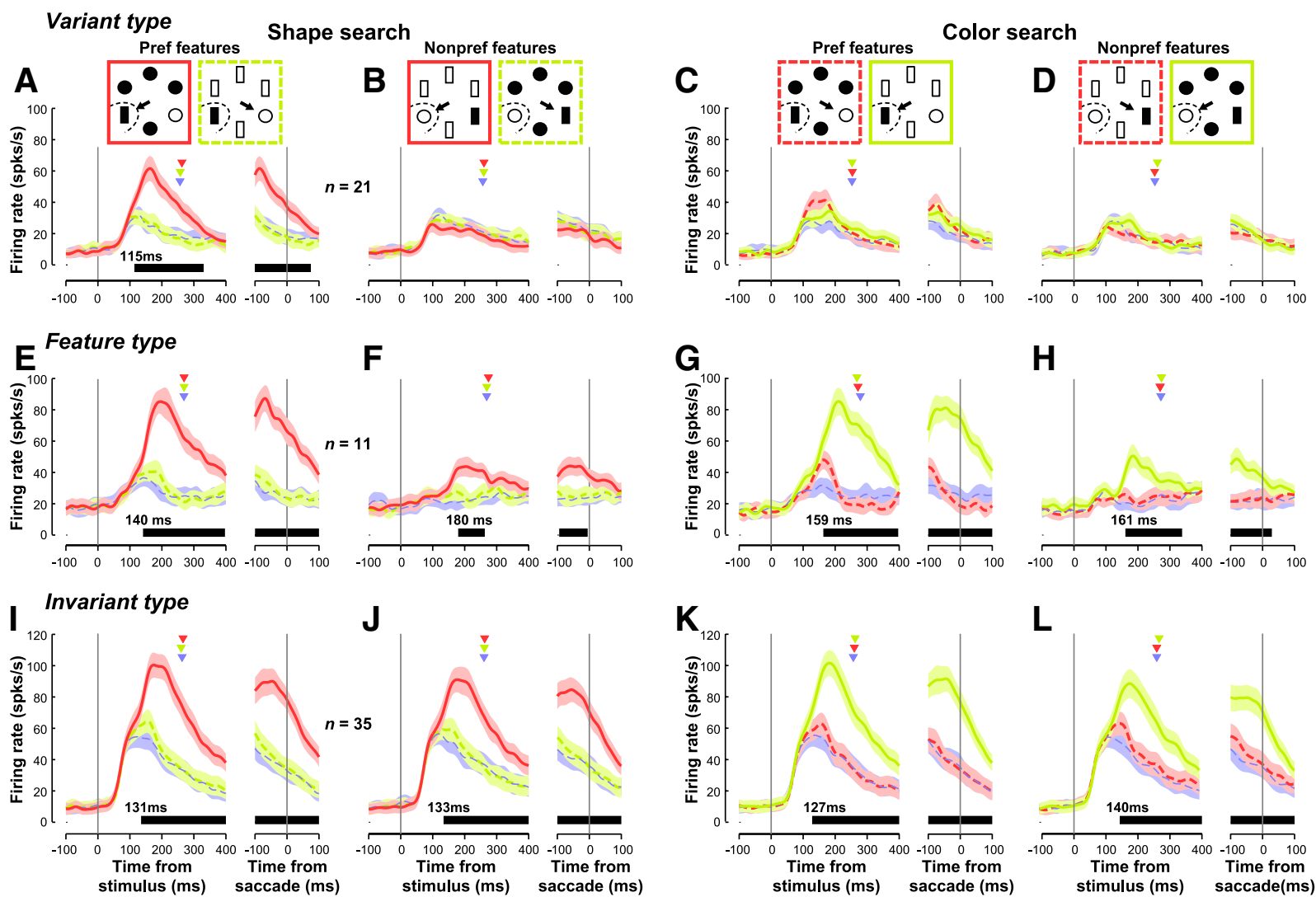

FIG. 7. Time course of the population responses. Average population responses of the variant-type neurons $(n=21)$ in the shape search $(A, B)$ and the color search $(C, D)$. For clarity, only neurons that preferred the shape dimension are shown. The stimulus shown in the receptive field had either the preferred $(A, C)$ or nonpreferred $(B, D)$ stimulus features. Traces in each panel are aligned at the time of array presentation (left) or saccade initiation (right). Thick solid, thick dashed, and thin dotted lines indicate the population responses to the target, distractor, and nontarget stimuli, respectively. Red, green, and blue lines respectively correspond to the responses to a shape, color, and nonsingleton stimuli. Shaded areas indicate 1SE. Red, green, and blue triangles respectively denote mean saccade reaction times when a shape, color, or nonsingleton stimulus was presented within the receptive field. Horizontal black lines below the spike density functions indicate the period during which the target was significantly discriminated from the distractor and nontarget stimuli (permutation test, $P<0.01$ ). The number above this horizontal line indicates the first time after the stimulus onset that target discrimination became significant and continued for $\geq 50$ ms. $E-H$ : time courses of the population responses of the feature-type neurons $(n=11)$ are shown in the same format as in $A-D$. I-L: time course of the population responses of the invariant-type neurons $(n=35)$.

When the stimulus features of the target were changed to the nonpreferred ones (Fig. $7 F$, red trace; Fig. $7 H$, green trace), the magnitude of the increase in activity elicited by the target was substantially diminished, but the average population responses still significantly discriminated the target from the other stimuli (permutation test, $P<0.01$ ), around $160-180 \mathrm{~ms}$ after array presentation. Figure 7, $I-L$ shows activity averaged across the invariant-type neurons $(n=35)$. Irrespective of the stimulus features and the target-defining dimension, the target discrimination became significant around $130-140 \mathrm{~ms}$ after array presentation (permutation test, $P<0.01$ ) and remained significant throughout the trials. Thus the increase in activity elicited by the target was not transient but tended to be sustained until saccade initiation. The differences in the response profiles of the three neuron types persisted over the course of visual saccade selection.

\section{Activity in error trials}

As shown in Fig. 6, $A$ and $B$, the variant-type neurons enhanced activities only when the target stimulus defined in the preferred dimension appeared in the receptive field and the monkey made a saccade to it. Data from only the correct trials were analyzed in these previous figures. It is interesting to know whether this enhanced activity was still induced even in the error trials in which the monkey incorrectly made a saccade to other stimuli outside the receptive field. To answer this question, we compared the activity in the correct and error trials (Fig. 8). Figure $8 A$ shows the activity of the same neuron as depicted in Fig. 3. This neuron preferred the target defined in the shape dimension. In all three conditions, the preferred shape singleton appeared in the receptive field. We found that the average activity during the error trials in the shape search (orange trace) was significantly greater than that during the correct trials in the color search (gray trace) (Mann-Whitney $U$ test; $P<0.01$ ), although in both conditions the monkey made a saccade to a stimulus outside the receptive field. On the other hand, the activity during the error trials in the shape search (orange trace) was almost the same as that during the correct trials in the shape search (black traces) (Mann-Whitney $U$ test; $P>0.1$, even though the saccades were very different between the two conditions.

We examined the activity on error trials for six variant-type neurons in which the monkeys made more than three errors when that target appeared in the receptive field. All six neurons preferred the shape singleton target in the shape search. Re- 

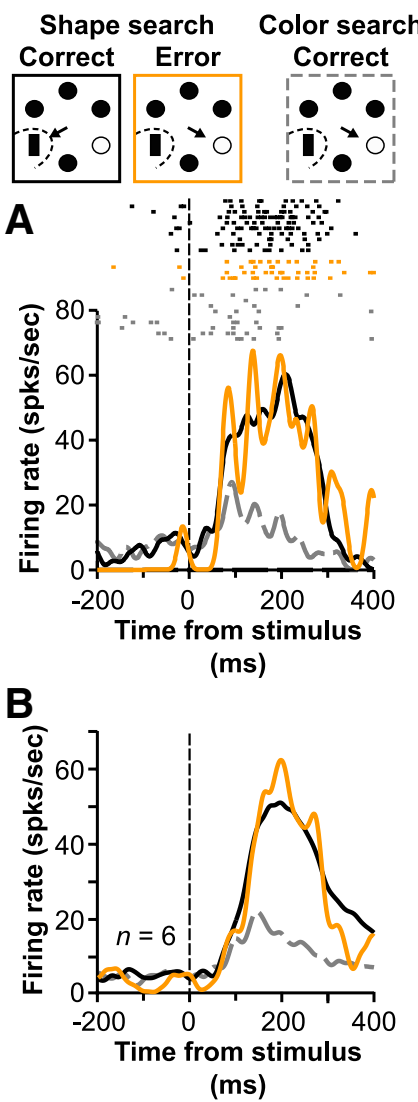

FIG. 8. Analysis of error trials. A: analysis of the responses in error trials in an example neuron that is the same as shown in Fig. 3. This neuron preferred the shape singleton target. The activities in 2 types of correct trials and one type of error trials are compared. In all 3 conditions, an identical array was presented in which the shape singleton (preferred stimulus) appeared in the receptive field and the color singleton (nonpreferred stimulus) appeared outside it. Black and gray traces, respectively, represent the correct trials in which the monkey made a saccade to the shape singleton target in the shape search and those in which it made a saccade to the color singleton target in the color search. Orange trace represents the error trials in which the monkey incorrectly made a saccade to the color singleton or nonsingleton stimulus in the shape search. Traces are aligned at the onset of the stimulus array. Top insets indicate the stimulus conditions and the behaviors. It should be noted that the activities during the error trials in the shape search (orange trace) were almost the same as those during the correct trials in the shape search (black trace). $B$ : population analysis. The average of activities of 6 variant-type neurons is shown in the same format as in A. All 6 neurons preferred the shape singleton target in the shape search and the monkeys made $>3$ errors when that target appeared in the receptive field.

sponses of these neurons showed a tendency similar to that of the example neuron depicted in Fig. 8A. In four of the six neurons, the activity during error trials in the shape search (orange frame condition in Fig. 8, inset) was significantly larger than that during correct trials in the color search (gray frame condition) (Mann-Whitney $U$ test; $P<0.01$ ). For three of these four neurons, the activity was not significantly different between the error and correct trials in the shape search (orange and black frame conditions) (Mann-Whitney $U$ test; $P>0.1)$. Figure $8 B$ shows the population activity averaged across the six neurons. The average activity during error and success trials in the shape search (orange and black traces) were not significantly different (Mann-Whitney $U$ test, $P>$ 0.1 ), whereas the difference between the average activity during error trials in the shape search (orange trace) and that during correct trials in the color search (gray trace) were nearly significant (Mann-Whitney $U$ test, $P=0.031$ ). These results suggest that the activities of the variant-type neurons were largely determined by the visual stimulus and the ongoing search dimension. In contrast, the direction of the impeding saccade seems to have much smaller effects on the activity. However, the small number of error trials and neurons used in this analysis make this conclusion tentative.

\section{Delay-period and saccade-burst activities during the memory-guided saccade task}

It has been repeatedly reported that PPC neurons exhibit delay-period activity during memory-guided saccade tasks. In the present study, we recorded neural activity only from a region in which neurons exhibited strong delay-period activity. To know whether there are systematic relationships between the classification of the three types of neurons-variant-, feature-, and invariant-type neurons-and the manifestation of delay-period activity, we recorded the activity of 51 neurons during both a multidimensional visual search task and a memory-guided saccade task. Of these, 18 neurons were variant type, 8 were feature type, 22 were invariant type, and 3 were none of those types. Among the first three groups, the proportions of neurons exhibiting delay-period activity were 10/18 (56\%), 5/8 (63\%), and 13/22 (59\%), respectively, which did not differ significantly $\left(\chi^{2}=0.61 ; \mathrm{df}=2 ; P>0.1\right)$; that is, we found no significant differences in the manifestation of delayperiod activity among the three main types of neurons.

We also examined the saccade-burst activity during the memory-guided saccade task. The respective proportions of neurons exhibiting saccade-burst activity among the variant, feature, and invariant types were $1 / 18(6 \%), 0 / 8(0 \%)$, and 9/22 (41\%). Thus a subset of the invariant-type neurons exhibited significant additional activity around the saccade onset, although virtually no additional activity was observed in the variant- or feature-type neurons.

\section{Dependence on shape and color features}

Previous studies reported the presence of neurons with selectivity for shape and color features in the PPC (Constantinidis and Steinmetz 2001; Lehky and Sereno 2007; Sereno and Amador 2006; Sereno and Maunsell 1998; Toth and Assad 2002). We also observed neural modulation that was dependent on shape and color in the present study. One advantage of our task design is that the dependencies on shape and color features can be evaluated in the same neurons. To do so, we calculated shape and color preference indices using the responses obtained under the eight target conditions. Because the responses to the target varied markedly between the two search conditions, each of the indices was computed separately for each of the search conditions (see METHODS).

Figure 9, $A$ and $B$ shows, respectively, the distributions of shape and color indices calculated for the 23 variant-type neurons. The dependence of the activity on the shape feature was evident (Fig. 9A). Among the 23 neurons, the value of the shape index was significantly different from 0 for 20 neurons (permutation analysis, $P<0.01$ ): 14 in the shape search, 0 in the color search, and 6 under both search conditions (Fig. 9A, filled black and gray circles). By contrast, the activity of 


\section{Variant type}
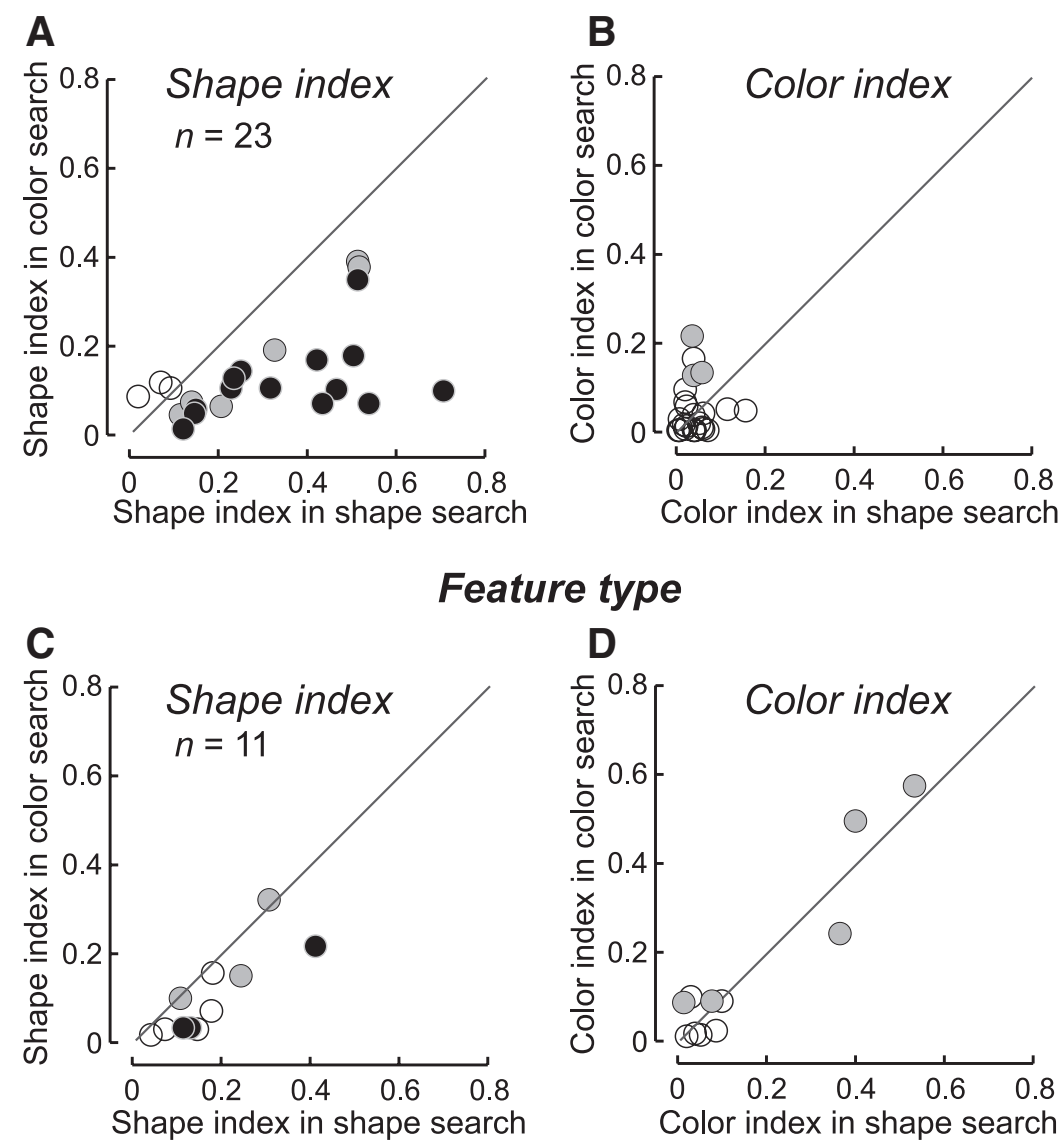

Feature type

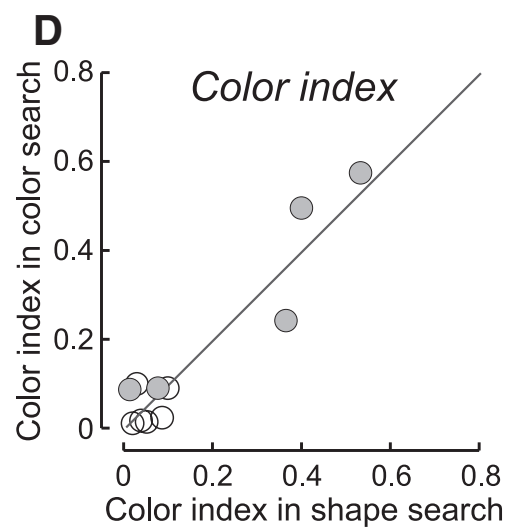

FIG. 9. Dependence on stimulus features. $A$ and $B$ : dependence of the activity of variant-type neurons $(n=23)$ on the shape feature $(A)$ and the color feature $(B)$ was examined. Values of each of the indices were separately calculated for the shape search (abscissa) and color search (ordinate). Open symbols indicate that the values of the shape or color indices were not significantly different from 0 (permutation test, $P>0.01$ ) under either search condition, whereas the black and gray symbols indicate that they were significantly different from 0 (permutation test, $P<0.01$ ) under the shape and/or color search conditions. Black symbols further indicate that the values of each of the indices significantly differed between the shape and color search conditions. $C$ and $D$ : shape and color indices for the feature-type neurons $(n=11) . E$ and $F$ : shape and color indices for the invariant-type neurons $(n=35)$.

\section{Invariant type}
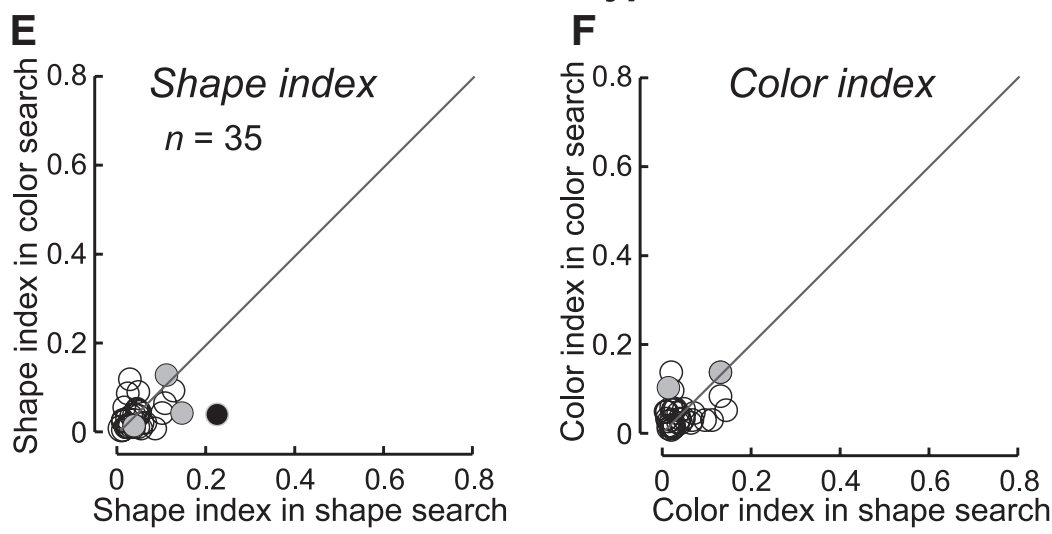

variant-type neurons showed little or no dependence on the color feature (Fig. 9B). The value of the color index was significant (permutation analysis, $P<0.01$ ) for only 3 neurons in the color search (Fig. $9 B$, filled gray circles). Further, only 2 neurons showed significance for both shape and color indices (permutation analysis, $P<0.01$ ). For one neuron, the value of the shape index was significant in the shape search and that of the color index was significant in the color search. For the other neuron, the value of the shape index was significant in both search conditions and the value of the color index was significant in the color search. Thus the number of neurons with a significant color feature preference was considerably smaller than the number with a significant shape feature preference.
Deviation of the data points from the diagonal line in the bottom-right direction indicates that the value of the shape index was larger in the shape search than in the color search, whereas deviation in the top-left direction indicates the opposite. Of 23 variant-type neurons, 14 exhibited significant modulation of the shape index between the two search conditions (permutation analysis, $P<0.01$ ) (Fig. 9A, filled black circles), suggesting that the strength of the shape dependence was modified as a function of the ongoing target-defining dimension (Sereno and Amador 2006; Toth and Assad 2002). By contrast, no neuron showed a significant change in the color index between the two search conditions (permutation analysis, $P>0.01)$ (Fig. 9B). 
Figure 9, $C$ and $D$ shows the results from the 11 feature-type neurons. Among those, the value of the shape index was significant for 6 neurons (permutation analysis, $P<0.01$ ): 2 in the shape search, 0 in the color search, and 4 under both search conditions (Fig. 9C, filled black and gray circles). In addition, 3 neurons showed significant modulation of the shape index between the two search conditions (permutation analysis, $P<$ 0.01) (Fig. 9C, black circles). The value of the color index was significant for 5 neurons (permutation analysis, $P<0.01$ ): 0 in the shape search, 1 in the color search, and 4 under both search conditions (Fig. 9D, filled gray circles). None of these neurons showed significant modulation of the color index between the two search conditions (permutation analysis, $P>0.01$ ) (Fig. $9 D)$. Furthermore, only one neuron showed significance for both shape and color indices (permutation analysis, $P<0.01$ ). The value of the shape index was significant in both search conditions and the value of the color index was significant only in the color search condition.

Figure 9, $E$ and $F$ shows the results from the 35 invarianttype neurons. Among those, the value of the shape index was significant for 4 neurons (permutation analysis, $P<0.01$ ): 3 in the shape search, 0 in the color search, and 1 in both search conditions (Fig. 9E, filled black and gray circles). Only one of those neurons showed significant modulation of the shape index between the two search conditions (permutation analysis, $P<0.01$ ) (Fig. 9E, black circle). Values of the color index for 3 neurons were significantly different from 0 (permutation analysis, $P<0.01)$ : 2 in the shape search and 1 in the color search (Fig. 9E, filled gray circles). None of these neurons showed significant modulation in the color index between the two search conditions (permutation analysis, $P>0.01$ ) (Fig. $9 F)$. Further, no neuron showed significance for both shape and color indices (permutation analysis, $P>0.1$ ).

\section{Recording sites}

In our exploration of neuronal activity, microelectrode penetrations were spaced at approximately $0.5-\mathrm{mm}$ intervals in the rostrocaudal direction, along the lateral bank of the intraparietal sulcus (see METHODS). The recording sites in one hemisphere of monkey $Q$ were histologically verified and the locations of 30 task-related neurons in this monkey were rendered and plotted on the cortical surface (Fig. 10A, bottom). The variant-type neurons (open circles) appeared to be distributed more in the anterior part of the lateral bank, whereas the invariant-type neurons (filled black circles) were distributed in the posterior part (Fig. 10B). The mean values for distance in the anterior-posterior location differed significantly between the variant- and invariant-type neurons (Mann-Whitney $U$ test, $P<0.001$ ). A similar tendency in the distributions of these two types of neurons was observed in the locations of 35 neurons from one hemisphere of monkey L (Fig. 10C), although the difference between the two distributions was not significant (Mann-Whitney $U$ test, $P=0.067$ ). Because the number of neurons recorded from another hemisphere of monkey $\mathrm{L}$ was small $(n=8)$, we did not examine the relationship between recording location and neuron type for these neurons.

\section{I S C U S S I O N}

We found that the neural representation specifying the locus of a target showed a great deal of variability in PPC neurons.
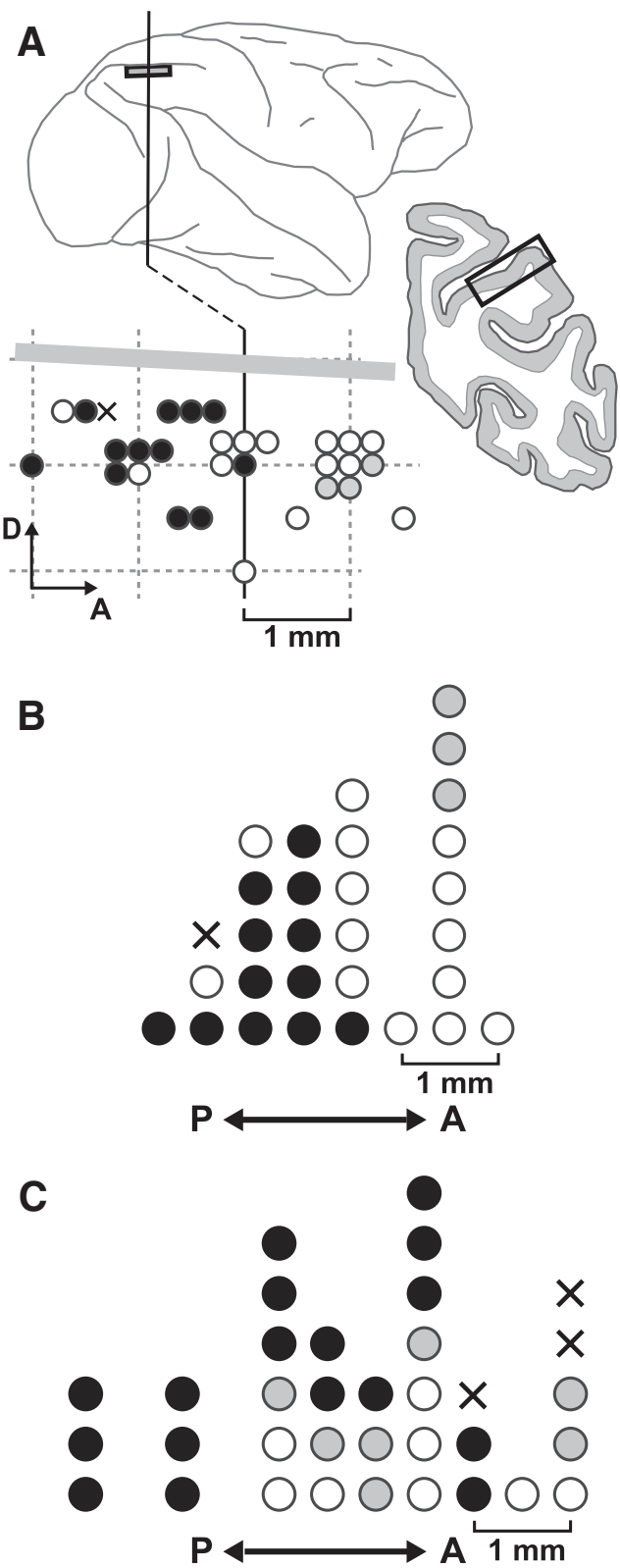

FIG. 10. Recording sites. A: schematic illustration of the recording sites in one monkey (monkey Q). The range of recording sites in the intraparietal sulcus is circumscribed by a thick solid line within the lateral view (top) and the coronal section drawing (right). The level of the coronal section is indicated by the vertical line in the lateral view. The location of each neuron is rendered on the cortical surface in the bottom panel. Neurons were sampled using a set of grids that had holes spaced $1.0 \mathrm{~mm}$ apart and were offset from each other by $0.5 \mathrm{~mm}$ : open circles, variant-type neurons; filled black circles, invariant-type neurons; filled gray circles, feature-type neurons; crosses, others. The thick gray line indicates the location of the intraparietal sulcus. $B$ and $C$ : anterior-posterior (AP) distribution of the 4 neuron types in the lateral bank of the intraparietal sulcus. The number of neurons obtained in each $0.5-\mathrm{mm}$ width is plotted in the AP direction: the data from monkey $\mathrm{Q}(B)$; the data from monkey $\mathrm{L}(C)$.

At one extreme was a subset of neurons that exhibited significantly enhanced activity only when the target had the preferred stimulus features and was defined in the preferred stimulus dimension (Fig. 6, $A$ and $B$, variant type). An intermediate subset of neurons showed strongly increased activity when the target had the preferred stimulus features (Fig. 6C, feature type). Finally, a subset of neurons at the other extreme 
invariably exhibited enhanced activity when the target was in the receptive field, irrespective of the stimulus features and target-defining dimension (Fig. 6D, invariant type). Such a wide variety of condition-dependent and condition-independent target selection processes suggests that the PPC may be one of the sites in the cortex where the transition from nonspatial- to spatial-based target selection processes takes place.

\section{Nonspatial target selection}

Neural activity related to target discrimination has been found in visual motor areas such as area $7 \mathrm{a}$ (Constantinidis and Steinmetz 2001), the LIP (Gottlieb et al. 1998; Ipata et al. 2006a; Thomas and Paré 2007), the frontal eye field (FEF) (Schall et al. 1995; Thompson et al. 1996), and the superior colliculus (Basso and Wurtz 1997; McPeek and Keller 2002). Generally, neural activity in those areas tends to be greater when the stimulus is behaviorally relevant than when it is irrelevant. We also found that about half of the PPC neurons studied exhibited enhanced activity when the target stimulus appeared in the receptive field, irrespective of the stimulus features, singleton type, or target-defining dimension (Fig. 6D, invariant type). A similar invariant target selection was found in the FEF (Ogawa and Komastu 2006). This spatial-based target selection is consistent with the hypothesis that PPC neurons represent the locus of spatial attention (Balan and Gottlieb 2006; Bisley and Goldberg 2003; Bushnell et al. 1981; Ipata et al. 2006a) or mediate signaling related to motor planning (Bracewell et al. 1996; Mazzoni et al. 1996; Snyder et al. 1997).

On the other hand, we also showed that in one third of PPC neurons (Fig. 6, $A$ and $B$, variant type), target discrimination was strongly regulated by both the stimulus features and the target-defining dimension. Even if the target had the preferred stimulus features and saccadic eye movements were made to it, if the target-defining dimension was not the preferred dimension, a significant increase in activity did not occur, indicating that this type of target discrimination cannot be explained by either spatial attention or motor planning. This was confirmed by results of the four-way ANOVA (i.e., a significant interaction was found among search dimension, behavioral relevance, and shape factors in the activity of variant-type neurons; Table 1). Thus the present findings suggest that the PPC could play a role not only in spatial-based target selection but also in nonspatial-based target selection.

\section{Representation of stimulus features in the PPC}

Previously reported findings suggest the PPC likely receives a variety of visual information from a number of visual areas via direct and indirect projections (Andersen et al. 1990; Asanuma et al. 1985; Borra et al. 2008) and is able to directly encode stimulus features (Constantinidis and Steinmetz 2001; Murata et al. 2000; Sereno and Amador 2006; Sereno and Maunsell 1998). Our identification of feature-type neurons (Fig. 6C) is consistent with that idea. In addition, neural representations of stimulus features can be altered as a function of task demand (Sereno and Amador 2006; Toth and Assad 2002) and we found similar modulation of feature preference in activity that was dependent on the ongoing search dimension (Fig. 6, $A$ and $B$ ). Moreover, we also found that although the task difficulty was nearly the same under the two search conditions, a subset of PPC neurons exhibited a significant preference for shape features, which suggests that even when the degrees of the behavioral relevance of the stimulus features are the same, this equivalence is not necessarily reflected in the activity of PPC neurons.

We found that the number of the variant-type neurons that significantly discriminated the target defined by the color dimension was considerably smaller than the number that significantly discriminated the target defined by the shape dimension (Fig. 6, $A$ and $B$ ). By contrast, invariant-type neurons significantly discriminated the target from the other stimuli, irrespective of whether the target was defined by the shape or the color dimension (Fig. 6D). This raises the question: How do invariant-type neurons receive information about the target defined by the color dimension? One possibility is that information about a target defined by the color dimension might be directly signaled from visual areas to invariant-type neurons but not variant-type neurons. Indeed, an earlier study demonstrated that the activity of a substantial population of V4 neurons can represent the location of a color singleton (Ogawa and Komatsu 2004), which suggests that signals from visual areas might be available to identify the location of a color singleton target. Another possibility is that variant-type neurons with color preference are widely and strongly connected to the invarianttype neurons. If that is the case, the widespread connections would permit the many invariant-type neurons to discriminate the target on the basis of color information, even though the population of the variant-type neurons with color preference is small. Through training, it may be possible to form such specially organized neural circuits in the LIP because LIP neurons exhibit strong learning-based plasticity in visual representation (Freedman and Assad 2006).

\section{Representation of visual saliency in the PPC}

Previous studies have shown that PPC neurons can specify the locations of intrinsically salient stimuli. For instance, responses in area $7 \mathrm{a}$ to a stimulus in the receptive field were greater when it was a singleton than when it was not, irrespective of the behavioral relevance of the singleton stimulus (Constantinidis and Steinmetz 2001, 2005). In addition, LIP neurons have been shown to represent visual salience associated with abrupt onset or temporal changes in basic stimulus features, even when they are behaviorally irrelevant (Balan and Gottlieb 2006; Bisley and Goldberg 2003). We also found that the activity of some variant-type neurons (5/23) was significantly increased when one specific singleton type appeared in the receptive field, irrespective of its behavioral relevance (Fig. 6, $A$ and $B$ ). A similar representation of intrinsic visual salience was found in visual area V4 when monkeys performed the same visual search task (Ogawa and Komatsu 2004). In the present study, however, we found that the activity of more than half of the variant-type neurons (18/23) specified the location of a singleton stimulus only when it was relevant in one particular search dimension, suggesting that neural representation of visual salience is strongly modulated by a dimensionspecific, top-down influence. Although such an influence on the representation of visual salience was also found in area V4, most V4 neurons tend to maintain their preference for a shape or color singleton stimuli across both search conditions (Ogawa and Komatsu 2004), indicating that the strength of the 
top-down influence is weaker in area V4 than that in the PPC. Thus although information about visual salience is reflected by neural modulation in both area V4 and the PPC, there might be a substantial difference in the strength of the top-down modulation in the two regions.

\section{Role of the PPC in visual selection}

In theoretical models of visual search or attention (Hamker 2004; Itti and Koch 2000; Koch and Ullman 1985; Wolfe 1994), information about stimulus features is separately computed in parallel within a set of feature-specific, retinotopically organized maps during an early stage of visual processing. The activities within these individual maps are then merged at a later stage to produce activity representing the importance of individual stimuli at each retinal location (saliency map). In these models, the variant- and feature-type neurons would correspond to early-stage processing (nonspatial target selection), whereas the invariant-type neurons would correspond to late-stage processing (spatial-based target selection). Thus from a theoretical perspective the PPC may include neurons that belong to different levels in the hierarchy of targetselection processing.

The present findings provide insight into how the PPC may function to execute correct responses in a given cognitive behavioral task. The activity of the variant-type neurons (Fig. $6, A$ and $B$ ) discriminates the target only when the target has specific stimulus features and is defined in one particular stimulus dimension. In addition, the activity of the feature-type neurons (Fig. 6C) discriminates the target only when it has specific stimulus features. These types of neurons can give partial solutions to the problem in the present visual search task. By contrast, the activity of the invariant-type neurons (Fig. 6D) always exhibits significant target discrimination, irrespective of the stimulus features or the target-defining dimension. Thus invariant-type neurons can give a complete solution to the present task problem. Moreover, their activity can be produced by simply summing the activities of the other two neuron types (Koch and Ullman 1985). This suggests that the brain may break down problems into multiple simpler subproblems and integrate partial solutions to these subproblems to obtain a complete solution to the original problem. This strategy is essentially the same as the "divide-and-conquer strategy," a powerful algorithm-design paradigm in computer science, which can work very efficiently in a parallel/distributed computing machine, such as the brain. The present findings may thus provide a concrete demonstration of the roles played by PPC neurons when solving a problem associated with a given cognitive task, such as a visual search task.

\section{A C K N O W L E D G M E N T S}

We thank K. Kawano and M. Yoshida for valuable comments on a preliminary version of this manuscript and M. Togawa and I. Yokoi for technical assistance.

\section{G R A N T S}

This work was supported by grants from the Japan Society for the Promotion of Science and by a Grant-in-Aid for Scientific Research on Priority Areas "Emergence of Adaptive Motor Function through Interaction between Body, Brain and Environment" from the Japanese Ministry of Education, Culture, Sports, Science, and Technology.

\section{REFERENCES}

Andersen RA, Asanuma C, Essick G, Siegel RM. Corticocortical connections of anatomically and physiologically defined subdivisions within the inferior parietal lobule. J Comp Neurol 296: 65-113, 1990.

Asanuma C, Andersen RA, Cowan WM. The thalamic relations of the caudal inferior parietal lobule and the lateral prefrontal cortex in monkeys: divergent cortical projections from cell clusters in the medial pulvinar nucleus. J Comp Neurol 241: 357-381, 1985.

Balan PF, Gottlieb J. Integration of exogenous input into a dynamic salience map revealed by perturbing attention. J Neurosci 26: 9239-9249, 2006.

Barash S, Bracewell RM, Fogassi L, Gnadt JW, Andersen RA. Saccaderelated activity in the lateral intraparietal area. I. Temporal properties; comparison with area 7a. J Neurophysiol 66: 1095-1108, 1991a.

Barash S, Bracewell RM, Fogassi L, Gnadt JW, Andersen RA. Saccaderelated activity in the lateral intraparietal area. II. Spatial properties. J Neurophysiol 66: 1109-1124, 1991b.

Basso MA, Wurtz RH. Modulation of neuronal activity by target uncertainty. Nature 389: 66-69, 1997.

Ben Hamed S, Duhamel JR, Bremmer F, Graf W. Visual receptive field modulation in the lateral intraparietal area during attentive fixation and free gaze. Cereb Cortex 12: 234-245, 2002.

Bichot NP, Rossi AF, Desimone R. Parallel and serial neural mechanisms for visual search in macaque area V4. Science 308: 529-534, 2005.

Bisley JW, Goldberg ME. Neuronal activity in the lateral intraparietal area and spatial attention. Science 299: 81-86, 2003.

Bisley JW, Krishna BS, Goldberg ME. A rapid and precise on-response in posterior parietal cortex. J Neurosci 24: 1833-1838, 2004.

Borra E, Belmalih A, Calzavara R, Gerbella M, Murata A, Rozzi S, Luppino G. Cortical connections of the macaque anterior intraparietal (AIP) area. Cereb Cortex 18: 1094-1111, 2008.

Bracewell RM, Mazzoni P, Barash S, Andersen RA. Motor intention activity in the macaque's lateral intraparietal area. II. Changes of motor plan. J Neurophysiol 76: 1457-1464, 1996.

Buschman TJ, Miller EK. Top-down versus bottom-up control of attention in the prefrontal and posterior parietal cortices. Science 315: 1860-1862, 2007.

Bushnell MC, Goldberg ME, Robinson DL. Behavioral enhancement of visual responses in monkey cerebral cortex. I. Modulation in posterior parietal cortex related to selective visual attention. J Neurophysiol 46: 755-772, 1981.

Colby CL, Duhamel JR, Goldberg ME. Visual, presaccadic, and cognitive activation of single neurons in monkey lateral intraparietal area. $J$ Neurophysiol 76: 2841-2852, 1996.

Constantinidis C, Steinmetz MA. Neuronal responses in area 7a to multiplestimulus displays: I. Neurons encode the location of the salient stimulus. Cereb Cortex 11: 581-591, 2001.

Constantinidis C, Steinmetz MA. Posterior parietal cortex automatically encodes the location of salient stimuli. J Neurosci 25: 233-238, 2005.

Duhamel JR, Colby CL, Goldberg ME. The updating of the representation of visual space in parietal cortex by intended eye movements. Science 255: 90-92, 1992.

Durand JB, Nelissen K, Joly O, Wardak C, Todd JT, Norman JF, Janssen P, Vanduffel W, Orban GA. Anterior regions of monkey parietal cortex process visual 3D shape. Neuron 55: 493-505, 2007.

Efron B, Tibshirani R. An Introduction to the Bootstrap. New York: Chapman \& Hall, 1993.

Freedman DJ, Assad JA. Experience-dependent representation of visual categories in parietal cortex. Nature 443: 85-88, 2006.

Fuchs AF, Robinson DA. A method for measuring horizontal and vertical eye movement chronically in the monkey. J Appl Physiol 21: 1068-1070, 1966.

Gnadt JW, Andersen RA. Memory related motor planning activity in posterior parietal cortex of macaque. Exp Brain Res 70: 216-220, 1988.

Gottlieb JP, Kusunoki M, Goldberg ME. The representation of visual salience in monkey parietal cortex. Nature 391: 481-484, 1998.

Hamker FH. A dynamic model of how feature cues guide spatial attention. Vision Res 44: 501-521, 2004.

Hikosaka O, Wurtz RH. Visual and oculomotor functions of monkey substantia nigra pars reticulata. III. Memory-contingent visual and saccade responses. J Neurophysiol 49: 1268-1284, 1983.

Ipata AE, Gee AL, Gottlieb J, Bisley JW, Goldberg ME. LIP responses to a popout stimulus are reduced if it is overtly ignored. Nat Neurosci 9: 1071-1076, 2006a. 
Ipata AE, Gee AL, Gottlieb J, Goldberg ME, Bisley JW. Activity in the lateral intraparietal area predicts the goal and latency of saccades in a free-viewing visual search task. J Neurosci 26: 3656-3661, 2006 b.

Itti L, Koch C. A saliency-based search mechanism for overt and covert shifts of visual attention. Vision Res 40: 1489-1506, 2000.

Judge SJ, Richmond BJ, Chu FC. Implantation of magnetic search coils for measurement of eye position: an improved method. Vision Res 20: 535-538, 1980.

Koch C, Ullman S. Shifts in selective visual attention: towards the underlying neural circuitry. Hum Neurobiol 4: 219-227, 1985.

Kodaka Y, Mikami A, Kubota K. Neuronal activity in the frontal eye field of the monkey is modulated while attention is focused on to a stimulus in the peripheral visual field, irrespective of eye movement. Neurosci Res 28: 291-298, 1997.

Konen CS, Kastner S. Two hierarchically organized neural systems for object information in human visual cortex. Nat Neurosci 11: 224-231, 2008.

Lehky SR, Sereno AB. Comparison of shape encoding in primate dorsal and ventral visual pathways. J Neurophysiol 97: 307-319, 2007.

Mazzoni P, Bracewell RM, Barash S, Andersen RA. Motor intention activity in the macaque's lateral intraparietal area. I. Dissociation of motor plan from sensory memory. J Neurophysiol 76: 1439-1456, 1996.

McPeek RM, Keller EL. Saccade target selection in the superior colliculus during a visual search task. J Neurophysiol 88: 2019-2034, 2002.

Mohler CW, Goldberg ME, Wurtz RH. Visual receptive fields of frontal eye field neurons. Brain Res 61: 385-389, 1973.

Monosov IE, Trageser JC, Thompson KG. Measurements of simultaneously recorded spiking activity and local field potentials suggest that spatial selection emerges in the frontal eye field. Neuron 57: 614-625, 2008.

Moore T, Fallah M. Microstimulation of the frontal eye field and its effects on covert spatial attention. J Neurophysiol 91: 152-162, 2004.

Motter BC. Neural correlates of attentive selection for color or luminance in extrastriate area V4. J Neurosci 14: 2178-2189, 1994.

Mountcastle VB, Lynch JC, Georgopoulos A, Sakata H, Acuna C. Posterior parietal association cortex of the monkey: command functions for operations within extrapersonal space. J Neurophysiol 38: 871-908, 1975.

Murata A, Gallese V, Luppino G, Kaseda M, Sakata H. Selectivity for the shape, size, and orientation of objects for grasping in neurons of monkey parietal area AIP. J Neurophysiol 83: 2580-2601, 2000.

Ogawa T, Komatsu H. Target selection in area V4 during a multidimensional visual search task. J Neurosci 24: 6371-6382, 2004.
Ogawa T, Komatsu H. Neuronal dynamics of bottom-up and top-down processes in area V4 of macaque monkeys performing a visual search. Exp Brain Res 173: 1-13, 2006.

Peng X, Sereno ME, Silva AK, Lehky SR, Sereno AB. Shape selectivity in primate frontal eye field. J Neurophysiol 100: 796-814, 2008.

Pigarev IN, Rizzolatti G, Scandolara C. Neurons responding to visual stimuli in the frontal lobe of macaque monkeys. Neurosci Lett 12: 207-212, 1979.

Richmond BJ, Optican LM, Podell M, Spitzer H. Temporal encoding of two-dimensional patterns by single units in primate inferior temporal cortex. I. Response characteristics. J Neurophysiol 57: 132-146, 1987.

Sakata H, Taira M. Parietal control of hand action. Curr Opin Neurobiol 4: 847-856, 1994.

Sakata H, Taira M, Murata A, Mine S. Neural mechanisms of visual guidance of hand action in the parietal cortex of the monkey. Cereb Cortex 5: 429-438, 1995

Schall JD, Hanes DP, Thompson KG, King DJ. Saccade target selection in frontal eye field of macaque. I. Visual and premovement activation. J Neurosci 15: 6905-6918, 1995.

Sereno AB, Amador SC. Attention and memory-related responses of neurons in the lateral intraparietal area during spatial and shape-delayed match-tosample tasks. J Neurophysiol 95: 1078-1098, 2006.

Sereno AB, Maunsell JH. Shape selectivity in primate lateral intraparietal cortex. Nature 395: 500-503, 1998.

Snyder LH, Batista AP, Andersen RA. Coding of intention in the posterior parietal cortex. Nature 386: 167-170, 1997.

Thomas NW, Paré M. Temporal processing of saccade targets in parietal cortex area LIP during visual search. J Neurophysiol 97: 942-947, 2007.

Thompson KG, Bichot NP, Sato TR. Frontal eye field activity before visual search errors reveals the integration of bottom-up and top-down salience. J Neurophysiol 93: 337-351, 2005.

Thompson KG, Hanes DP, Bichot NP, Schall JD. Perceptual and motor processing stages identified in the activity of macaque frontal eye field neurons during visual search. J Neurophysiol 76: 4040-4055, 1996.

Toth LJ, Assad JA. Dynamic coding of behaviourally relevant stimuli in parietal cortex. Nature 415: 165-168, 2002.

Wardak C, Olivier E, Duhamel JR. Saccadic target selection deficits after lateral intraparietal area inactivation in monkeys. J Neurosci 22: 9877-9884, 2002.

Wolfe JM. Guided search 2.0: a revised model of visual search. Psychon Bull Rev 1: 202-238, 1994. 